\title{
A projection semi-implicit scheme for the coupling of an elastic structure with an incompressible fluid
}

\author{
M. A. Fernández, J.-F. Gerbeau* and C. Grandmont ${ }^{\dagger *}$
}

September 27, 2005

\begin{abstract}
We address the numerical simulation of fluid-structure systems involving an incompressible viscous fluid. This issue is particularly difficult to face when the fluid added-mass acting on the structure is strong, as it happens in hemodynamics for example. Indeed, several works have shown that, in such situations, implicit coupling seems to be necessary in order to avoid numerical instabilities. Although significant improvements have been achieved during the last years, solving implicit coupling often exhibits a prohibitive computational cost. In this work, we introduce a semi-implicit coupling scheme which remains stable for a reasonable range of the discretization parameters. The first idea consists in treating implicitly the added-mass effect, whereas the other contributions (geometrical non-linearities, viscous and convective effects) are treated explicitly. The second idea, relies on the fact that this kind of explicit-implicit splitting can be naturally performed using a Chorin-Temam projection scheme in the fluid. We prove (conditional) stability of the scheme for a fully discrete formulation. Several numerical experiments point out the efficiency of the present scheme compared to several implicit approaches.
\end{abstract}

keywords:fluid-structure interaction; semi-implicit coupling; added-mass effect; projection scheme; blood flows.

\section{INTRODUCTION}

We propose in this article a new method to solve fluid-structure interaction problems involving an incompressible viscous fluid. This coupling may be delicate when the so-called addedmass effect is strong. Indeed, in such situations, implicit coupling schemes (also known as strongly coupled), i.e. preserving energy balance, seems to be necessary to avoid numerical instabilities. This leads to very expensive simulations since, at each time step, a lot of resolutions of the fluid and the structure problems have to be performed. Nevertheless, in other situations, e.g. when dealing with compressible fluids or with a low added-mass effect,

*INRIA, Projet REO, Rocquencourt BP 105, F-78153 Le Chesnay Cedex, France

${ }^{\dagger}$ Université Paris Dauphine, Place du Maréchal de Lattre de Tassigny, F-75775 Paris, France 
explicit coupling schemes (also known as loosely or weakly coupled), i.e. typically requiring only one fluid and structure resolution per time step, prove to be stable. The purpose of this paper is to propose and analyze a partially explicit (or semi-implicit) scheme, which remains stable in test cases for which only fully implicit schemes were known to be stable (to the best of our knowledge). The proposed algorithm, based on the classical Chorin-Temam projection scheme for incompressible flows $[5,6,46]$, is much more efficient than any strongly coupled scheme we are aware of. Moreover, its stability properties, observed in various complex numerical simulations, are confirmed by a theoretical analysis carried out in a simplified configuration.

\section{MOTIVATIONS AND MAIN IDEAS}

To explain and motivate the proposed algorithm, a few notations are needed. Let $\Omega(t)$ be a time-dependent domain in $\mathbb{R}^{d}(d=2$ or 3$)$, see Figure 1 . We assume, for all time $t$, that $\overline{\Omega(t)}=\overline{\Omega^{f}(t)} \cup \overline{\Omega^{s}(t)}$ and $\Omega^{f}(t) \cap \Omega^{s}(t)=\emptyset$, where $\Omega^{f}(t)$ is occupied by an incompressible viscous fluid and $\Omega^{s}(t)$ by an elastic solid. The fluid-structure interface is denoted by $\Sigma(t)=$ $\overline{\Omega^{f}(t)} \cap \overline{\Omega^{s}(t)}$.

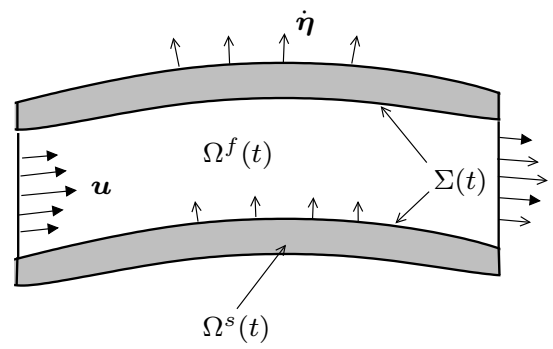

Figure 1: The current configuration $\Omega(t)$.

The velocity and the Cauchy stress tensor are respectively denoted by $\boldsymbol{u}$ and $\boldsymbol{\sigma}^{f}$ in the fluid and by $\dot{\boldsymbol{\eta}}$ and $\boldsymbol{\sigma}^{s}$ in the structure. Before discretization, the fluid-structure coupling is defined through the transmission conditions

$$
\boldsymbol{u}=\dot{\boldsymbol{\eta}}, \quad \boldsymbol{\sigma}^{f} \cdot \boldsymbol{n}^{f}+\boldsymbol{\sigma}^{s} \cdot \boldsymbol{n}^{s}=0, \quad \text { on } \quad \Sigma,
$$

where $\boldsymbol{n}^{f}$ (resp. $\boldsymbol{n}^{s}$ ) denote the outward normal on $\partial \Omega^{f}$ (resp. $\partial \Omega^{s}$ ).

From the computational viewpoint, a straightforward way to satisfy the discrete counterpart of (1) is to simultaneously solve the fluid and the structure problems in a unique solver. This results in the so-called monolithic or direct methods. Examples of such an approach are too numerous to be reviewed extensively: for instance, we can cite recent works using an Arbitrary Lagrangian Euler formulation [45, 31], a fictitious domain method [8, 1] or transpiration interface boundary conditions [23]. By construction, a monolithic method enforces the transmission relation (1): one says that it is strongly coupled. As a consequence, 
a monolithic method is generally stable in the energy norm. But this approach needs ad hoc software development and typically results in a global solver which is less modular than two distinct fluid and structure solvers. In particular it is difficult to devise efficient global preconditionners, and to maintain state-of-the-art schemes in each solver.

With the so-called partitioned procedures, the fluid and the structure are solved with two different codes. This allows the use of "legacy software", and increases the capabilities of evolution and optimization of each code. In fluid-structure problems this idea goes back, at least, to the work by K.C. Park. One of the historical motivations of his Staggered Solution Procedure is recalled by C.A. Felippa in [17]: in 1975, the US Office of Naval Research awarded a contract for the development of a 3D "underwater shock" code with the constraint, set by submarine-builders, of using NASTRAN. But this code had become a proprietary program and the source code was no more accessible. Thus, the use of monolithic methods was ruled out. Of course, code accessibility is not the only motivation for the staggered procedures: an attractive feature of this strategy is also the possibility of using for each sub-problem the methods the most adapted to its specific mathematical properties. Nevertheless, it soon appeared that too naive staggered schemes are limited by a very small time step. Much work has then been dedicated to the improvement of this basic idea. For example, tricky predictors to improve accuracy [43, 42], or the augmentation concept to relax time step constraints (e.g. [18]).

Among the partitioned schemes, we have to distinguish the weakly coupled ones from the strongly coupled. A scheme is said to be weakly (or loosely) coupled when (1) is not exactly satisfied at each time step, or in other words, when a spurious numerical power may appear on the fluid-structure interface. Let us emphasize that a partitioned scheme is not necessarily weakly coupled: when sub-iterations are performed at each time step, the transmission conditions (1) can be enforced with a high accuracy even though two different solvers are used. Nevertheless, partitioned procedures are often used to implement weakly coupled schemes. Indeed, many fluid-structure interaction problems, in particular in aeroelasticity, can be solved in practice without enforcing exactly (1). We will not address here those situations which have been widely studied in the literature. We refer for example to [16] for a state-of-the-art presentation of such approaches.

In the present work, we are interested in those cases where the classical weakly coupled schemes are apparently unstable, as observed in various numerical studies (for instance [38, $32,40,26]$ ). This typically occurs in the internal flow of an incompressible fluid whose density is close to the structure density. Blood flows provide an example of such a situation. A few papers have proposed partial theoretical explanations of this fact. The role of the added-mass effect has been pointed out in [32]. A stability criterion, involving the density ratio and a geometrical parameter, has been established in [3]. It is worth noticing that this theoretical criterion is independent of the time step, which confirms empirical observations: reducing the time step does not cure these instabilities. Let us mention that the incompressibility of the fluid seems to play an important role in the observed instabilities. This may be related to the fact that, in the case of an incompressible fluid, the partitioned algorithm is applied to a system of differential algebraic equations, as pointed out in [34, 35]. 
To our knowledge, strongly coupled schemes have been, up to now, the only way of avoiding those numerical instabilities. To enforce transmission conditions (1) in a partitioned approach, many sub-iterations have to be performed, at each time step, which often leads to prohibitive computational costs. This explains why, these last years, so many works have been devoted to the development of efficient methods for the solution of the non-linear problems arising in implicit coupling.

A number of fixed point strategies have been proposed: in [32] a steepest-descent algorithm is presented, an Aitken-like acceleration formula in used in [39, 37] and transpiration boundary conditions are used in [11] to avoid the computation of the fluid matrices at each sub-iteration. In [44], the authors propose to relax the fluid incompressibility constraint in order to accelerate the convergence of the fixed-point iterations. The artificial compressibility is devised to eventually vanish at convergence. This tricky idea outlines the importance of the divergence-free constraint in the coupled problem.

Further advances on the topic suggested the use of Newton based methods for a fast convergence towards the solution of the coupled non-linear system. These methods require the evaluation of the Jacobian associated to the fluid-solid coupled state equations. Inexact Newton methods are addressed in $[33,35,47,31]$ by using finite difference approximations of the Jacobian, in $[47,48,25,26,31]$ by replacing the coupled tangent operator by a simpler linear operator and, applied to complex geometries in [27]. Exact Newton algorithms, including differentiation with respect to the fluid domain, are introduced in $[20,21,19,22]$, see also [41].

Acceleration techniques using Krylov spaces have been proposed in [12, 31, 36]. The heterogeneous domain decomposition point of view adopted in [32], and further developed in [10], offers an interesting unified presentation of all these partitioned strongly coupled schemes. Note, in particular, that most of the coupling algorithms are based on the so-called Dirichlet-Neumann strategy, which means that the fluid is solved by enforcing the velocity, and the structure by enforcing the load. A nonlinear extension of the so-called NeumannNeumann strategy has been proposed in $[9,10]$.

Although significant improvements have been achieved over the last years, none of the existing strategies are able, in the test cases we are interested in and at least to our knowledge, to circumvent strong coupling without compromising stability.

The method that we propose and analyze in this paper is not strongly coupled, in the sense that (1) is not exactly enforced, but it exhibits very good stability properties. It basically relies upon two ideas. The first one is to couple implicitly the pressure stress to ensure stability. This is suggested in [3] where it is shown that explicit coupling of the added mass term yields instabilities. The remaining terms of the fluid equations - dissipation, convection and geometrical non-linearities - are explicitly coupled to the structure (of course, these terms may be implicit within the fluid solver). This drastically reduces the cost of the coupling without affecting too much the stability. The second idea, relies upon the fact that this kind of implicit-explicit splitting can be conveniently performed using a Chorin-Temam projection scheme (see for instance [29]) in the fluid: at each time step we propose to strongly couple the projection sub-step (carried out in a known fluid domain) with the structure, so accounting 
for the added-mass effect in an implicit way, while the expensive ALE-advection-viscous substep is explicitly, i.e. weakly, coupled. The main advantages of the resulting algorithm are: its simplicity of implementation (specially compared to sophisticated Newton-like methods) and its efficiency compared to any strongly coupled schemes we are aware of. Obviously, the main drawbacks are: first, it assumes the fluid to be solved with a projection scheme and, second, the energy is not perfectly balanced, at least from a theoretical viewpoint. In spite of that, theoretical and numerical evidence show that, for a wide range of physical and discrete parameters, the scheme is numerically stable.

The remainder of the paper is organized as follows. In the next Section we introduce a general fluid-structure interaction problem and describe its associated mathematical model. In Section 4, the resulting system of equations is semi-discretized in time using the new coupling scheme based on the Chorin-Temam projection method. In Section 5, we provide a theoretical stability analysis of our scheme on a simplified linear model problem. The numerical results are presented in Section 6, showing the relevance and efficiency of the proposed scheme. Finally, we give some conclusions and draw some lines for a future work. 


\section{PROBLEM SETTING}

Let $\hat{\Omega}=\hat{\Omega}^{f} \cup \hat{\Omega}^{s}$ be a reference configuration of the system, see Figure 2. We denote the deformation of the solid medium by

$$
\varphi^{s}: \hat{\Omega}^{s} \times[0, T] \longrightarrow \Omega^{s}(t)
$$

We then introduce the corresponding deformation gradient $\boldsymbol{F}^{s}(\hat{x}, t) \stackrel{\text { def }}{=} \boldsymbol{\nabla}_{\hat{x}} \boldsymbol{\varphi}(\hat{x}, t)$, and its determinant $J^{s}(\hat{x}, t) \stackrel{\text { def }}{=} \operatorname{det} \boldsymbol{F}^{s}(\hat{x}, t)$. The displacement of the domain is given by $\boldsymbol{\eta}(\hat{x}, t) \stackrel{\text { def }}{=}$ $\boldsymbol{\varphi}^{s}(\hat{x}, t)-\hat{x}$. Within the structure, the velocity of a material point $\hat{x}, \partial_{t} \boldsymbol{\varphi}^{s}(\hat{x}, t)=\partial_{t} \boldsymbol{\eta}(\hat{x}, t)$, is denoted by $\dot{\boldsymbol{\eta}}$. Within the fluid, $\boldsymbol{u}(x, t)$ stands for the fluid velocity at a point $x$ of the fluid domain. In addition, we adopt a fluid Arbitrary Lagrangian Eulerian (ALE) formulation by introducing another mapping,

$$
A: \hat{\Omega}^{f} \times[0, T] \longrightarrow \Omega^{f}(t),
$$

such that $A_{\mid \hat{\Sigma}}=\varphi_{\mid \hat{\Sigma}}^{s}$, but which in general does not follows the material trajectories (see [13], for instance). We then introduce its corresponding deformation gradient $\boldsymbol{F}^{f}(\hat{x}, t) \stackrel{\text { def }}{=}$ $\boldsymbol{\nabla}_{\hat{x}} A(\hat{x}, t)$, and determinant $J^{f}(\hat{x}, t) \stackrel{\operatorname{def}}{=} \operatorname{det} \boldsymbol{F}^{f}(\hat{x}, t)$. The fluid domain velocity is denoted by $\boldsymbol{w}(\hat{x}, t) \stackrel{\text { def }}{=} \partial_{t} A(\hat{x}, t)$.
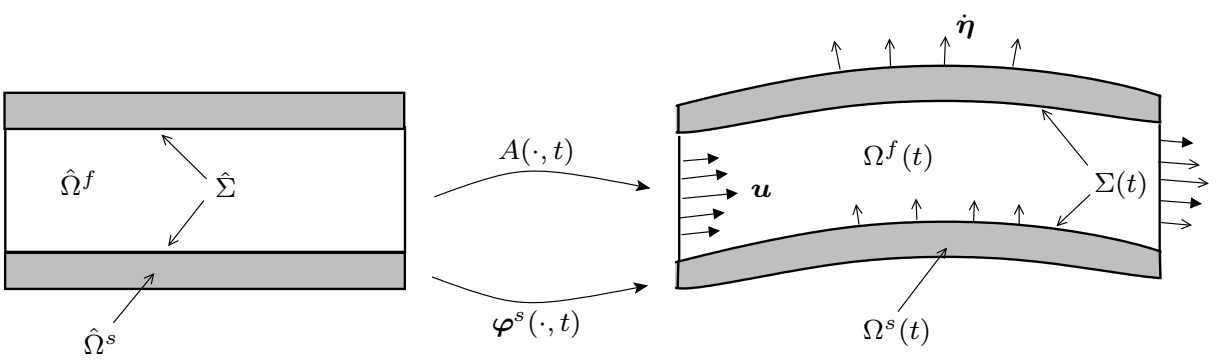

Figure 2: Parametrization of the domains $\Omega^{f}(t)$ and $\Omega^{s}(t)$.

We introduce the first Piola-Kirchhoff tensor in the structure

$$
\boldsymbol{\Pi} \stackrel{\text { def }}{=} J^{s}\left(\boldsymbol{\sigma}^{s} \circ \varphi^{s}\right)\left(\boldsymbol{F}^{s}\right)^{-T} .
$$

The field $\boldsymbol{\Pi}$ is related to $\boldsymbol{\eta}$ through an appropriate constitutive law. We assume the fluid to be incompressible and Newtonian, thus

$$
\boldsymbol{\sigma}^{f}(\boldsymbol{u}, p) \stackrel{\text { def }}{=}-p \mathbf{I}+2 \mu \boldsymbol{\epsilon}(\boldsymbol{u}),
$$

with $p$ the pressure and

$$
\boldsymbol{\epsilon}(\boldsymbol{u}) \stackrel{\text { def }}{=} \frac{1}{2}\left(\boldsymbol{\nabla} \boldsymbol{u}+\boldsymbol{\nabla} \boldsymbol{u}^{T}\right),
$$


the strain rate tensor.

The fluid-structure problem then reads:

$$
\begin{aligned}
& \left\{\begin{aligned}
\boldsymbol{w}=\operatorname{Ext}\left(\left.\dot{\boldsymbol{\eta}}\right|_{\hat{\Sigma}}\right), & \text { in } \quad \hat{\Omega}^{f}, \\
\left.\rho^{f} \frac{\partial \boldsymbol{u}}{\partial t}\right|_{\hat{x}}+\rho^{f}(\boldsymbol{u}-\boldsymbol{w}) \cdot \nabla \boldsymbol{u}-2 \mu \operatorname{div}(\boldsymbol{\epsilon}(\boldsymbol{u}))+\nabla p=0, & \text { in } \quad \Omega^{f}(t), \\
\operatorname{div} \boldsymbol{u}=0, & \text { in } \quad \Omega^{f}(t), \\
\boldsymbol{u}=\dot{\boldsymbol{\eta}} \circ\left(\boldsymbol{\varphi}^{s}\right)^{-1}, & \text { on } \Sigma(t),
\end{aligned}\right. \\
& \left\{\begin{aligned}
\rho^{s} \frac{\partial^{2} \boldsymbol{\eta}}{\partial t^{2}}-\operatorname{div}_{\hat{x}}(\boldsymbol{\Pi})=0, & \text { in } \quad \hat{\Omega}^{s}, \\
\boldsymbol{\Pi} \cdot \boldsymbol{n}^{s}=J^{f} \boldsymbol{\sigma}^{f}(\boldsymbol{u}, p) \cdot\left(\boldsymbol{F}^{f}\right)^{-T} \cdot \boldsymbol{n}^{s}, & \text { on } \quad \hat{\Sigma}
\end{aligned}\right.
\end{aligned}
$$

where $\left.\frac{\partial}{\partial t}\right|_{\hat{x}}$ represents the ALE time derivative and Ext denotes any reasonable extension operator in the fluid domain. Of course, the above coupled system has to be complemented with adequate boundary conditions on $\partial \Omega(t)$, that will depend on the physical problem under consideration.

\section{A PROJECTION BASED SEMI-IMPLICIT COUPLING}

We present in this section our semi-implicit coupling scheme. We assume that the incompressible Navier-Stokes equations are solved with the Chorin-Temam projection scheme and that the structure equations are discretized with the mid-point rule. We denote by $\delta t$ the time step, and, for the sake of clarity we present the time semi-discrete version of the algorithm. Assuming that $\Omega^{n}, \boldsymbol{u}^{n}, p^{n}, \boldsymbol{\eta}^{n}$ are known at time $t^{n}$, we propose to compute $\Omega^{n+1}, \boldsymbol{u}^{n+1}$, $p^{n+1}, \boldsymbol{\eta}^{n+1}$ according to the following procedure:

- Step 0: Second order extrapolation of the fluid-structure interface:

$$
\tilde{\boldsymbol{\eta}}^{n+1}=\boldsymbol{\eta}^{n}+\delta t\left(\frac{3}{2} \dot{\boldsymbol{\eta}}^{n}-\frac{1}{2} \dot{\boldsymbol{\eta}}^{n-1}\right) .
$$

- Step 1: Definition of the new domain:

$$
\boldsymbol{w}_{\mid \hat{\Sigma}}^{n+1}=\frac{\tilde{\boldsymbol{\eta}}^{n+1}-\boldsymbol{\eta}^{n}}{\delta t} \mid \hat{\Sigma}, \quad \boldsymbol{w}^{n+1}=\operatorname{Ext}\left(\boldsymbol{w}_{\mid \Sigma}^{n+1}\right), \quad \Omega^{f, n+1}=\Omega^{f, n}+\delta t \boldsymbol{w}^{n+1} .
$$

- Step 2: ALE-advection-diffusion step (explicit coupling):

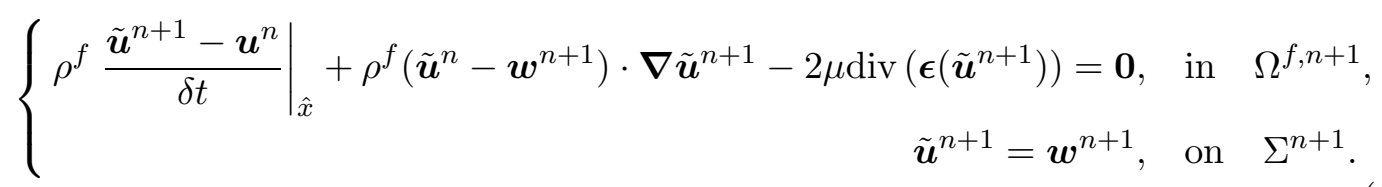


- Step 3: Projection step (implicit coupling):

- Step 3.1:

$$
\left\{\begin{array}{rrr}
\rho^{f} \frac{\boldsymbol{u}^{n+1}-\tilde{\boldsymbol{u}}^{n+1}}{\delta t}+\boldsymbol{\nabla} p^{n+1}=\mathbf{0}, & \text { in } & \Omega^{f, n+1} \\
\operatorname{div} \boldsymbol{u}^{n+1}=0, & \text { in } & \Omega^{f, n+1} \\
\boldsymbol{u}^{n+1} \cdot \boldsymbol{n}^{f}=\frac{\boldsymbol{\eta}^{n+1}-\boldsymbol{\eta}^{n}}{\delta t} \cdot \boldsymbol{n}^{f}, & \text { on } & \Sigma^{n+1}
\end{array}\right.
$$

- Step 3.2:

$$
\left\{\begin{array}{rrr}
\rho^{s} \frac{\dot{\boldsymbol{\eta}}^{n+1}-\dot{\boldsymbol{\eta}}^{n}}{\delta t}-\operatorname{div} \hat{x}\left(\frac{\boldsymbol{\Pi}^{n}+\boldsymbol{\Pi}^{n+1}}{2}\right)=\mathbf{0}, & \text { in } \quad \hat{\Omega}^{s}, \\
\frac{\boldsymbol{\eta}^{n+1}-\boldsymbol{\eta}^{n}}{\delta t}=\frac{\dot{\boldsymbol{\eta}}^{n+1}+\dot{\boldsymbol{\eta}}^{n}}{2}, & \text { in } \quad \hat{\Omega}^{s}, \\
\boldsymbol{\Pi}^{n+1} \cdot \boldsymbol{n}^{s}=J^{f, n+1}\left(\boldsymbol{\sigma}^{f}\left(\tilde{\boldsymbol{u}}^{n+1}, p^{n+1}\right) \circ A^{n+1}\right)\left(\boldsymbol{F}^{f, n+1}\right)^{-T} \cdot \boldsymbol{n}^{s}, & \text { on } & \hat{\Sigma} .
\end{array}\right.
$$

Note that the steps 1 and 2 are performed only once per time step. In a partitioned procedure, step 3 is solved by sub-iterating between steps 3.1 and 3.2 (using fixed-point or Newton iterations, for instance) since $\boldsymbol{\eta}^{n+1}$ is required in 3.1 while $p^{n+1}$ is required in 3.2. The two sub-problems of steps 3 are therefore solved several times but contrarily to a fully coupled procedure, the part of the fluid solved during the inner iterations reduces to a simple Darcylike problem. In a standard strongly coupled approach, the domain velocity in step 1 would be defined from the (unknown) solution of the structure problem by

$$
\boldsymbol{w}_{\mid \hat{\Sigma}}^{n+1}=\frac{\boldsymbol{\eta}^{n+1}-\boldsymbol{\eta}^{n}}{\delta t} \mid \hat{\Sigma} \cdot
$$

The sub-iterations would therefore include step 1 and step 2 which increases dramatically the overall computational cost. The main contribution of this work is to show that steps 1 and 2 can indeed be treated "outside" the inner loop of sub-iterations, without compromising too much the stability.

Remark 1 A variant of the above scheme can be obtained by switching steps 2 and 3, i.e. using a velocity-correction scheme within the fluid [29].

\section{STABILITY ANALYSIS}

In this section we analyze the stability of a slightly simplified version of our semi-implicit coupling scheme, when applied to a linear model problem. 


\subsection{A simplified coupled problem}

We consider a low Reynolds regime and assume that the interface undergoes infinitesimal displacements. The fluid might then be simply described by the Stokes equations in a fixed domain $\Omega^{f} \subset \mathbb{R}^{d}, d=2,3$. For the structure we also consider a linear behavior, described either by the classical linear elastodynamics equations or by equations based on linear beam/shell models. The reference domain of the solid is denoted by $\Omega^{s} \subset \mathbb{R}^{d}$. It will be either a domain or a surface of $\mathbb{R}^{d}$ (in this later case the elastic domain is identified to its mid-surface). We still denote by $\Sigma \stackrel{\text { def }}{=} \partial \Omega^{s} \cap \partial \Omega^{f}$ the fluid-structure interface. In the case the structure is described by beam/shell model we have $\overline{\Omega^{s}}=\Sigma$, see Figure 3 .

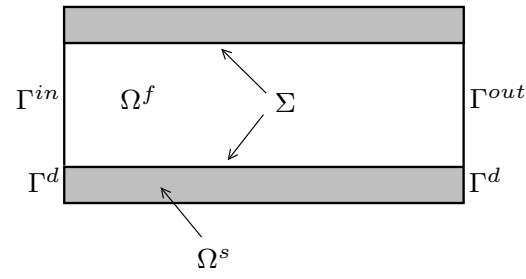

(a) Case $\overline{\Omega^{s}} \neq \Sigma$

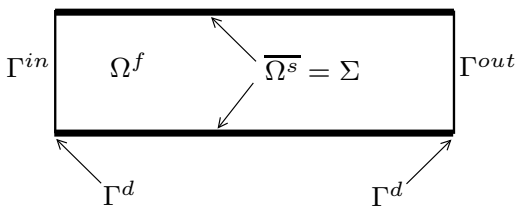

(b) Case $\overline{\Omega^{s}}=\Sigma$

Figure 3: Examples of geometric configurations.

In particular, when dealing with a $d$-dimensional linear structure, our simplified coupled problem reads as follows:

$$
\begin{aligned}
& \left\{\begin{array}{lr}
\rho^{f} \partial_{t} \boldsymbol{u}-2 \mu \operatorname{div}(\boldsymbol{\epsilon}(\boldsymbol{u}))+\nabla p=\mathbf{0}, & \text { in } \Omega^{f}, \\
\operatorname{div} \boldsymbol{u}=0, & \text { in } \Omega^{f}, \\
\boldsymbol{u}=\partial_{t} \boldsymbol{\eta}, & \text { on } \Sigma, \\
\boldsymbol{\sigma}^{f}(\boldsymbol{u}, p) \cdot \boldsymbol{n}^{f}=\mathbf{0}, & \text { on } \quad \Gamma^{\text {in }} \cup \Gamma^{\text {out }} .
\end{array}\right. \\
& \left\{\begin{array}{lr}
\rho^{s} \partial_{t t} \boldsymbol{\eta}-\nabla \cdot \boldsymbol{\sigma}^{s}(\boldsymbol{\eta})=\mathbf{0}, & \text { in } \Omega^{s}, \\
\boldsymbol{\sigma}^{s}(\boldsymbol{\eta}) \cdot \boldsymbol{n}^{s}=\boldsymbol{\sigma}^{f}(\boldsymbol{u}, p) \cdot \boldsymbol{n}^{s}, & \text { on } \Sigma, \\
\boldsymbol{\eta}=\mathbf{0}, & \text { on } \Gamma^{d} .
\end{array}\right.
\end{aligned}
$$

Here, $\boldsymbol{\sigma}^{s}(\boldsymbol{\eta})$ stands for the linearized Cauchy stress tensor, related to displacement $\boldsymbol{\eta}$ through an appropriate constitutive law (Hook's law, for instance).

In the sequel, we will consider the usual Sobolev spaces $H^{m}(\Omega), m \geq 0$, for a given domain $\Omega \subset \mathbb{R}^{d}$. In particular, we have $L^{2}(\Omega)=H^{0}(\Omega)$. The scalar product in $L^{2}(\Omega)$ is denoted by $(\cdot, \cdot)_{\Omega}$ and its norm by $\|\cdot\|_{0, \Omega}$.

In order to provide a variational setting for the above coupled problem, we define the 
following spaces of test functions:

$$
V^{f} \stackrel{\text { def }}{=}\left\{\boldsymbol{v}^{f} \in\left[H^{1}\left(\Omega^{f}\right)\right]^{d}: \quad \boldsymbol{v}^{f}=0 \quad \text { on } \quad \Sigma\right\},
$$

and in the case of the $d$-dimensional linearized elasticity

$$
V^{s} \stackrel{\text { def }}{=}\left\{\boldsymbol{v}^{s} \in\left[H^{1}\left(\Omega^{s}\right)\right]^{d}: \quad \boldsymbol{v}^{s}=0 \quad \text { on } \quad \Gamma^{d}\right\} .
$$

Multiplying the fluid equation (9) by $\boldsymbol{v}^{f}$ and the solid equations by $\boldsymbol{v}^{s}$, integrating by parts and taking into account the boundary conditions, it follows that any regular solution of (9)-(10) satisfies the following weak formulation: for all $\left(\boldsymbol{v}^{f}, q\right) \in V^{f} \times L^{2}\left(\Omega^{f}\right)$ and $\boldsymbol{v}^{s} \in V^{s}$

$$
\left\{\begin{array}{l}
\rho^{f}\left(\partial_{t} \boldsymbol{u}, \boldsymbol{v}^{f}\right)_{\Omega^{f}}+2 \mu\left(\boldsymbol{\epsilon}(\boldsymbol{u}), \boldsymbol{\epsilon}\left(\boldsymbol{v}^{f}\right)\right)_{\Omega^{f}}-\left(p, \nabla \cdot \boldsymbol{v}^{f}\right)_{\Omega^{f}}+(q, \nabla \cdot \boldsymbol{u})_{\Omega^{f}}=0 \\
\boldsymbol{u}=\partial_{t} \boldsymbol{\eta}, \quad \text { on } \quad \Sigma, \\
\rho^{s}\left(\partial_{t t} \boldsymbol{\eta}, \boldsymbol{v}^{s}\right)_{\Omega^{s}}+a^{s}\left(\boldsymbol{\eta}, \boldsymbol{v}^{s}\right)=-\left\langle\mathcal{R}(\boldsymbol{u}, p), \mathcal{L}\left(\boldsymbol{v}^{s}\right)\right\rangle
\end{array}\right.
$$

where the operator $\mathcal{L}$ represents a continuous extension operator from $V^{s}$ into the space

$$
W^{f} \stackrel{\text { def }}{=}\left\{\boldsymbol{v}^{f} \in\left[H^{1}\left(\Omega^{f}\right)\right]^{d}: \quad \boldsymbol{v}^{f}=0 \quad \text { on } \quad \Gamma^{\text {in }} \cup \Gamma^{o u t}\right\} .
$$

Here, we used the notation:

$$
a^{s}\left(\boldsymbol{\eta}, \boldsymbol{v}^{s}\right) \stackrel{\text { def }}{=}\left(\boldsymbol{\sigma}^{s}(\boldsymbol{\eta}), \boldsymbol{\epsilon}\left(\boldsymbol{v}^{s}\right)\right)_{\Omega^{s}} .
$$

Finally, the load on the structure is given by the following duality pair

$$
\left\langle\mathcal{R}(\boldsymbol{u}, p), \boldsymbol{v}^{f}\right\rangle \stackrel{\text { def }}{=}\left(\partial_{t} \boldsymbol{u}, \boldsymbol{v}^{f}\right)_{\Omega^{f}}+2 \mu\left(\boldsymbol{\epsilon}(\boldsymbol{u}), \boldsymbol{\epsilon}\left(\boldsymbol{v}^{f}\right)\right)_{\Omega^{f}}-\left(p, \nabla \cdot \boldsymbol{v}^{f}\right)_{\Omega^{f}}, \quad \forall \boldsymbol{v}^{f} \in W^{f} .
$$

This expression represents the residual of the variational fluid sub-problem $(11)_{1}$ when tested with functions that do not vanish on $\Sigma$. It can be readily checked that, for any solution $(\boldsymbol{u}, p)$ of $(11)_{1}$, we have

$$
\left\langle\mathcal{R}(\boldsymbol{u}, p), \mathcal{L}\left(\boldsymbol{v}^{s}\right)\right\rangle=\int_{\Sigma} \boldsymbol{\sigma}^{f}(\boldsymbol{u}, p) \boldsymbol{n}^{f} \cdot \boldsymbol{v}^{s} .
$$

However, computing the fluid interface load from the variational fluid residual has the major advantage of still making sense after discretization in space, and to lead to somewhat more stable numerical results.

When dealing with beam or shell models the weak formulation takes a similar form, but with different definitions of $a^{s}$ and $V^{s}$ depending on the considered structure model. Thus, in the sequel, $a^{s}$ will stand for a general symmetric bi-linear form, coercive on $V^{s}$, which describes the linear elastic behavior of the solid. Moreover, we assume the space $V^{s}$ to be continuously embedded in $\left[H^{1}\left(\Omega^{s}\right)\right]^{d}$. 
Remark 2 By testing (11) with $\boldsymbol{v}^{f}=\boldsymbol{u}-\mathcal{L}\left(\partial_{t} \boldsymbol{\eta}\right), q=p$ and $\boldsymbol{v}^{s}=\partial_{t} \boldsymbol{\eta}, \quad\left(\boldsymbol{v}^{f}\right.$ is admissible thanks to $\left.(11)_{2}\right)$, one readily obtains the following energy equality:

$$
\frac{\mathrm{d}}{\mathrm{d} t}\left[\frac{\rho^{f}}{2}\|\boldsymbol{u}\|_{0, \Omega}^{2}+\frac{\rho^{s}}{2}\left\|\partial_{t} \boldsymbol{\eta}\right\|_{0, \Omega}^{2}+\frac{1}{2} a^{s}(\boldsymbol{\eta}, \boldsymbol{\eta})\right]+2 \mu\|\boldsymbol{\epsilon}(\boldsymbol{u})\|_{0, \Omega}^{2}=0 .
$$

In other words, the variation of the mechanical energy of the system is equal to the power dissipated by the fluid viscosity.

In the next paragraph we address the time discretization of (11) using a simplified version of the semi-implicit projection scheme introduced in Section 4.

\subsection{Time semi-discretization}

In order to set up the variational formulation of the projection sub-step, we introduce the following spaces:

$$
Y^{f} \stackrel{\text { def }}{=}\left\{\boldsymbol{v}^{f} \in\left[L^{2}\left(\Omega^{f}\right)\right]^{d}: \nabla \cdot \boldsymbol{v}^{f} \in L^{2}\left(\Omega^{f}\right)\right\}, \quad Y_{0}^{f} \stackrel{\text { def }}{=}\left\{\boldsymbol{v}^{f} \in Y^{f}: \boldsymbol{v}^{f} \cdot \boldsymbol{n}^{f}=0 \quad \text { on } \quad \Sigma\right\} .
$$

Assuming that $\tilde{\boldsymbol{u}}^{n}, \boldsymbol{u}^{n}, p^{n}$ and $\boldsymbol{\eta}^{n}$ are known, the time semi-discretized formulation we will analyze writes: find $\left(\tilde{\boldsymbol{u}}^{n+1}, \boldsymbol{u}^{n+1}, p^{n+1}, \boldsymbol{\eta}^{n+1}\right) \in\left[H^{1}\left(\Omega^{f}\right)\right]^{d} \times Y^{f} \times L^{2}\left(\Omega^{f}\right) \times V^{s}$ such that:

- Diffusion step (explicit coupling):

$$
\left\{\begin{array}{l}
\frac{\rho^{f}}{\delta t}\left(\tilde{\boldsymbol{u}}^{n+1}-\boldsymbol{u}^{n}, \tilde{\boldsymbol{v}}^{f}\right)_{\Omega^{f}}+2 \mu\left(\boldsymbol{\epsilon}\left(\tilde{\boldsymbol{u}}^{n+1}\right), \boldsymbol{\epsilon}\left(\tilde{\boldsymbol{v}}^{f}\right)\right)_{\Omega^{f}}=0, \quad \forall \tilde{\boldsymbol{v}}^{f} \in V^{f}, \\
\tilde{\boldsymbol{u}}^{n+1}=\frac{\boldsymbol{\eta}^{n}-\boldsymbol{\eta}^{n-1}}{\delta t}, \quad \text { on } \quad \Sigma,
\end{array}\right.
$$

- Projection step (implicit coupling)

$$
\left\{\begin{array}{l}
\frac{\rho^{f}}{\delta t}\left(\boldsymbol{u}^{n+1}-\tilde{\boldsymbol{u}}^{n+1}, \boldsymbol{v}^{f}\right)_{\Omega^{f}}-\left(p^{n+1}, \nabla \cdot \boldsymbol{v}^{f}\right)_{\Omega^{f}}+\left(q, \nabla \cdot \boldsymbol{u}^{n+1}\right)_{\Omega^{f}}=0, \\
\forall\left(\boldsymbol{v}^{f}, q\right) \in Y_{0}^{f} \times L^{2}\left(\Omega^{f}\right), \\
\boldsymbol{u}^{n+1} \cdot \boldsymbol{n}^{f}=\frac{\boldsymbol{\eta}^{n+1}-\boldsymbol{\eta}^{n}}{\delta t} \cdot \boldsymbol{n}^{f}, \quad \text { on } \quad \Sigma, \\
\frac{\rho^{s}}{\delta t^{2}}\left(\boldsymbol{\eta}^{n+1}-2 \boldsymbol{\eta}^{n}+\boldsymbol{\eta}^{n-1}, \boldsymbol{v}^{s}\right)_{\Omega^{s}}+a^{s}\left(\boldsymbol{\eta}^{n+1}, \boldsymbol{v}^{s}\right)=-\left\langle\mathcal{R}_{\mu}\left(\tilde{\boldsymbol{u}}^{n+1}\right), \mathcal{L}\left(\boldsymbol{v}^{s}\right)\right\rangle \\
-\left\langle\mathcal{R}_{p}\left(\boldsymbol{u}^{n+1}, p^{n+1}\right), \mathcal{L}\left(\boldsymbol{v}^{s}\right)\right\rangle, \quad \forall \boldsymbol{v}^{s} \in V_{0}^{s},
\end{array}\right.
$$


with the following definition of the fluid residuals $\mathcal{R}_{\mu}$ and $\mathcal{R}_{p}$ :

$$
\begin{aligned}
\left\langle\mathcal{R}_{\mu}\left(\tilde{\boldsymbol{u}}^{n+1}\right), \tilde{\boldsymbol{v}}^{f}\right\rangle & \stackrel{\text { def }}{=} \frac{\rho^{f}}{\delta t}\left(\tilde{\boldsymbol{u}}^{n+1}-\boldsymbol{u}^{n}, \tilde{\boldsymbol{v}}^{f}\right)_{\Omega^{f}}+2 \mu\left(\boldsymbol{\epsilon}\left(\tilde{\boldsymbol{u}}^{n+1}\right), \boldsymbol{\epsilon}\left(\tilde{\boldsymbol{v}}^{f}\right)\right)_{\Omega^{f}}, \\
\left\langle\mathcal{R}_{p}\left(\boldsymbol{u}^{n+1}, p^{n+1}\right), \boldsymbol{v}^{f}\right\rangle & \stackrel{\text { def }}{=} \frac{\rho^{f}}{\delta t}\left(\boldsymbol{u}^{n+1}-\tilde{\boldsymbol{u}}^{n+1}, \boldsymbol{v}^{f}\right)_{\Omega^{f}}-\left(p^{n+1}, \nabla \cdot \boldsymbol{v}^{f}\right)_{\Omega^{f}},
\end{aligned}
$$

for all $\tilde{\boldsymbol{v}}^{f}, \boldsymbol{v}^{f} \in W^{f}$.

Remark 3 We have considered here a simplified version of the coupling scheme presented in Section 4. In particular, the fluid domain being fixed, no ALE terms appear in the equations. Moreover, for the sake of simplicity, we switch off the second order extrapolation step and assume that the solid equations are discretized in time with a leap-frog scheme. Nevertheless, the main feature of the coupling scheme is preserved: the diffusion step is explicitly coupled with the structure.

The above scheme involves a splitting of the residual $\mathcal{R}$ in terms of the residuals $\mathcal{R}_{\mu}$ and $\mathcal{R}_{p}$. The former represents the interface fluid viscous stress (explicitly treated), whereas the later corresponds to the interface fluid pressure stress (implicitly treated).

In the next paragraph we introduce a finite element discretization of (13)-(14).

\subsection{Space discretization: fully discrete problem}

Let $\left\{\mathcal{T}_{h}^{f}\right\}_{0<h \leq 1}\left(\left\{\mathcal{T}_{H}^{s}\right\}_{0<H \leq 1}\right)$ a family of triangulations of the domains $\Omega^{f}$ (resp. $\Omega^{s}$ ) satisfying the usual requirements of finite element approximations. For each triangulation the subscripts $h, H \in(0,1]$ refer to the level of refinement of the triangulation. In particular, $h$ is defined as

$$
h \stackrel{\text { def }}{=} \max _{K \in \mathcal{T}_{h}} h_{K},
$$

with $h_{K}$ the diameter of the element $K$. We define $H$ in an analogous way. In addition, we assume that both families of triangulations are quasi-uniform. For instance, for $\left\{\mathcal{T}_{h}^{f}\right\}_{0<h \leq 1}$, this implies that

$$
\frac{h_{K}}{\rho_{K}}<C_{R}, \quad h_{K} \geq C_{U} h, \quad \forall K \in \mathcal{T}_{h}, \quad \forall h \in(0,1]
$$

where $\rho_{K}$ stands for the diameter of the largest inscribed ball in $K$ and $C_{R}, C_{U}>0$ are fixed constants. In the sequel, $C>0$ is used to denote a generic mesh-independent constant.

We define $Q_{h}^{f}$ as an internal Lagrange finite element approximation of $L^{2}\left(\Omega^{f}\right), V_{h}^{f}$ as an internal Lagrange finite element approximation of $\left[H^{1}\left(\Omega^{f}\right)\right]^{d}, V_{H}^{s}$ being an internal finite element space approximation of $V^{s}$. We set $V_{h, 0}^{f} \stackrel{\text { def }}{=} V_{h}^{f} \cap V^{f}$. The space $Y^{f}$ (resp. $Y_{0}^{f}$ ) is approximated by $Y_{h}^{f}$ (resp. $Y_{h, 0}^{f}$ ) such that $V_{h}^{f} \subset Y_{h}^{f}$. For a discussion on the possible choices of approximation spaces for the projection method we refer to [30].

Remark 4 Solving the projection sub-step as a Darcy-like problem requires an inf-sup condition on the pair $\left(Q_{h}^{f}, V_{h}^{f}\right)$. However, this additional assumption does not play any role in the following stability analysis. 
We denote by $V_{H}^{s}(\Sigma)$ and $V_{h}^{f}(\Sigma)$ the trace finite element spaces associated to $V_{H}^{s}$ and $V_{h}^{f}$, respectively. Note that $V_{H}^{s}(\Sigma)$ is equal to $V_{H}^{s}$ whenever $\Omega^{s} \subset \mathbb{R}^{d-1}$. Finally, $\pi_{h}: V_{H}^{s}(\Sigma) \longrightarrow$ $V_{h}^{f}(\Sigma)$ stands for a given interface matching operator. For instance, $\pi_{h}$ can be a linear interpolation operator (nodal-wise matching) or a projection based operator (see [2]). For a discussion on the optimality of such choices we refer to [15, 28].

Therefore, the fully discretized problem writes as follows: find $\left(\tilde{\boldsymbol{u}}_{h}^{n+1}, \boldsymbol{u}_{h}^{n+1}, p_{h}^{n+1}, \boldsymbol{\eta}_{H}^{n+1}\right) \in$ $V_{h}^{f} \times Y_{h}^{f} \times Q_{h}^{f} \times V_{H}^{s}$ such that

$$
\begin{aligned}
& \left\{\begin{array}{l}
\frac{\rho^{f}}{\delta t}\left(\tilde{\boldsymbol{u}}_{h}^{n+1}-\boldsymbol{u}_{h}^{n}, \tilde{\boldsymbol{v}}_{h}^{f}\right)_{\Omega^{f}}+2 \mu\left(\boldsymbol{\epsilon}\left(\tilde{\boldsymbol{u}}_{h}^{n+1}\right), \boldsymbol{\epsilon}\left(\tilde{\boldsymbol{v}}_{h}^{f}\right)\right)_{\Omega^{f}}=0, \quad \forall \tilde{\boldsymbol{v}}_{h}^{f} \in V_{h, 0}^{f}, \\
\tilde{\boldsymbol{u}}_{h}^{n+1}=\pi_{h}\left(\frac{\boldsymbol{\eta}_{H}^{n}-\boldsymbol{\eta}_{H}^{n-1}}{\delta t}\right), \quad \text { on } \Sigma,
\end{array}\right. \\
& \left\{\begin{array}{l}
\frac{\rho^{f}}{\delta t}\left(\boldsymbol{u}_{h}^{n+1}-\tilde{\boldsymbol{u}}_{h}^{n+1}, \boldsymbol{v}_{h}^{f}\right)_{\Omega^{f}}-\left(p_{h}^{n+1}, \nabla \cdot \boldsymbol{v}_{h}^{f}\right)_{\Omega^{f}}+\left(q_{h}, \nabla \cdot \boldsymbol{u}_{h}^{n+1}\right)_{\Omega^{f}}=0, \\
\forall\left(\boldsymbol{v}_{h}^{f}, q_{h}\right) \in Y_{h, 0}^{f} \times Q_{h}^{f}, \\
\boldsymbol{u}_{h}^{n+1} \cdot \boldsymbol{n}^{f}=\pi_{h}\left(\frac{\boldsymbol{\eta}_{H}^{n}-\boldsymbol{\eta}_{H}^{n-1}}{\delta t}\right) \cdot \boldsymbol{n}^{f}, \quad \text { on } \quad \Sigma, \\
\frac{\rho^{s}}{\delta t^{2}}\left(\boldsymbol{\eta}_{H}^{n+1}-2 \boldsymbol{\eta}_{H}^{n}+\boldsymbol{\eta}_{H}^{n-1}, \boldsymbol{v}_{H}^{s}\right)_{\Omega^{s}}+a^{s}\left(\boldsymbol{\eta}_{H}^{n+1}, \boldsymbol{v}_{H}^{s}\right)=-\left\langle\mathcal{R}_{\mu}\left(\tilde{\boldsymbol{u}}_{h}^{n+1}\right), \mathcal{L}_{h}\left(\boldsymbol{v}_{H}^{s}\right)\right\rangle \\
-\left\langle\mathcal{R}_{p}\left(\boldsymbol{u}_{h}^{n+1}, p_{h}^{n+1}\right), \mathcal{L}_{h}\left(\boldsymbol{v}_{H}^{s}\right)\right\rangle, \quad \forall \boldsymbol{v}_{H}^{s} \in V_{H}^{s} .
\end{array}\right.
\end{aligned}
$$

Here, $\mathcal{L}_{h}: V_{H}^{s}(\Sigma) \longrightarrow V_{h}^{f}$ stands for a given discrete lifting operator satisfying

$$
\mathcal{L}_{h}\left(\boldsymbol{b}_{H}\right)=\pi_{h}\left(\boldsymbol{b}_{H}\right), \quad \text { on } \quad \Sigma \text {. }
$$

In particular, in the following, $\mathcal{L}_{h}$ will be defined by:

$$
\mathcal{L}_{h}\left(\boldsymbol{b}_{H}\right) \stackrel{\text { def }}{=} \sum_{\substack{i=1 \\ x_{h}^{i} \in \Sigma}}^{N_{h}} \pi_{h}\left(\boldsymbol{b}_{H}\right)\left(x_{h}^{i}\right) \varphi_{h}^{i},
$$

with $\left\{x_{h}^{i}\right\}_{i=1}^{N_{h}},\left\{\varphi_{h}^{i}\right\}_{i=1}^{N_{h}}$ the sets of nodes and shape functions of $V_{h}^{f}$. This choice is motivated by the fact that, under appropriate assumptions on $\pi_{h}, \mathcal{L}_{h}$ has suitable mathematical properties enabling us to prove the stability of (16)-(17) (see Lemma 1 below). Moreover, from the numerical point of view, (18) is indeed the discrete lifting operator used for computing the fluid residuals $\mathcal{R}_{\mu}$ and $\mathcal{R}_{p}$.

\subsection{Main stability result}

The following Theorem provides the conditional stability of the coupling scheme (16)-(17). 
Theorem 1 Assume that the interface matching operator $\pi_{h}: V_{H}^{s}(\Sigma) \longrightarrow V_{h}^{f}(\Sigma)$ is $L^{2}$ stable. Then, there exists a constant $C>0$, independent of the physical and discretization parameters, such that under the condition

$$
\rho^{s} \geq C\left(\rho^{f} \frac{h}{H^{\alpha}}+2 \frac{\mu \delta t}{h H^{\alpha}}\right), \quad \text { with } \quad \alpha \stackrel{\text { def }}{=} \begin{cases}0, & \text { if } \overline{\Omega^{s}}=\Sigma, \\ 1, & \text { if } \overline{\Omega^{s}} \neq \Sigma,\end{cases}
$$

the following discrete energy estimate holds:

$$
\begin{gathered}
\frac{1}{\delta t}\left[\frac{\rho^{f}}{2}\left\|\boldsymbol{u}_{h}^{n+1}\right\|_{0, \Omega^{f}}^{2}-\frac{\rho^{f}}{2}\left\|\boldsymbol{u}_{h}^{n}\right\|_{0, \Omega^{f}}^{2}\right]+\frac{1}{\delta t}\left[\frac{\rho^{s}}{2}\left\|\frac{\boldsymbol{\eta}_{H}^{n+1}-\boldsymbol{\eta}_{H}^{n}}{\delta t}\right\|_{0, \Omega^{f}}^{2}-\frac{\rho^{s}}{2}\left\|\frac{\boldsymbol{\eta}_{H}^{n}-\boldsymbol{\eta}_{H}^{n-1}}{\delta t}\right\|_{0, \Omega^{f}}^{2}\right] \\
+\frac{1}{2 \delta t}\left[a_{s}\left(\boldsymbol{\eta}_{H}^{n+1}, \boldsymbol{\eta}_{H}^{n+1}\right)-a_{s}\left(\boldsymbol{\eta}_{H}^{n}, \boldsymbol{\eta}_{H}^{n}\right)\right]+\mu\left\|\boldsymbol{\epsilon}\left(\tilde{\boldsymbol{u}}_{h}^{n+1}\right)\right\|_{0, \Omega^{f}}^{2} \leq 0 .
\end{gathered}
$$

Therefore, the semi-implicit coupling scheme (16)-(17) is stable, in the energy-norm, under condition (19).

Before giving the proof of the previous theorem, some remarks are in order:

- The assumption on the $L^{2}$-stability of the interface matching operator is satisfied by the standard finite element interpolation operator, for example, whenever the fluid interface triangulation is a sub-triangulation of the solid interface triangulation (see Lemma 2 in appendix). This includes, in particular, the case of interface matching meshes. By construction, a mortar based matching operator also fulfills that assumption (see [2]).

- The sufficient condition (19) can be satisfied by reducing the ratios $\frac{h}{H^{\alpha}}$ and $\frac{\delta t}{h H^{\alpha}}$. The later might be thought as a CFL-like condition.

- In the case $\overline{\Omega^{s}}=\Sigma$, i.e. $\alpha=0$, condition (19) becomes independent of the solid mesh size $H$. In particular, we may set $H=h$, and stabilize the scheme by simply reducing $h($ and $\delta t)$.

- In the case $\overline{\Omega^{s}} \neq \Sigma$, i.e. $\alpha=1$, the stability of the scheme can be ensured provided that the fluid mesh size $h$ is small enough compared to the structure mesh size $H$. Numerical simulations performed in $2 D$ and $3 D$, with $h=H$, showed however that this condition seems to be not necessary, when dealing with a reasonable range of physical parameters. Indeed, other sufficient stability conditions can be derived following the arguments of the proof below (see Remark 5).

Further discussions on the stability of the scheme are given at the end of this paragraph as a series of remarks.

Proof. In order to derive the discrete energy estimate, the first natural idea (see Remark 2) would consist in testing (16)-(17) with

$$
\boldsymbol{v}_{H}^{s}=\frac{\boldsymbol{\eta}_{H}^{n+1}-\boldsymbol{\eta}_{H}^{n}}{\delta t}, \quad \tilde{\boldsymbol{v}}_{h}^{f}=\tilde{\boldsymbol{u}}_{h}^{n+1}-\mathcal{L}_{h}\left(\boldsymbol{v}_{H}^{s}\right), \quad \boldsymbol{v}_{h}^{f}=\boldsymbol{u}_{h}^{n+1}-\mathcal{L}_{h}\left(\boldsymbol{v}_{H}^{s}\right), \quad q_{h}=p_{h}^{n+1} .
$$


However, due to the explicit coupling of the diffusion step, the trace $\left.\tilde{\boldsymbol{v}}_{h}^{f}\right|_{\Sigma}$ is not necessarily zero. In other words, $\tilde{\boldsymbol{v}}_{h}^{f}$ is not an admissible test function. This can be overcome by subtracting the appropriate correction term, which leads us to the choice:

$$
\begin{aligned}
& \boldsymbol{v}_{H}^{s}=\frac{\boldsymbol{\eta}_{H}^{n+1}-\boldsymbol{\eta}_{H}^{n}}{\delta t}, \quad \tilde{\boldsymbol{v}}_{h}^{f}=\tilde{\boldsymbol{u}}_{h}^{n+1}-\mathcal{L}_{h}\left(\boldsymbol{v}_{H}^{s}\right)-\mathcal{L}_{h}\left(\frac{\boldsymbol{\eta}_{H}^{n+1}-2 \boldsymbol{\eta}_{H}^{n}+\boldsymbol{\eta}_{H}^{n-1}}{\delta t}\right), \\
& \boldsymbol{v}_{h}^{f}=\boldsymbol{u}_{h}^{n+1}-\mathcal{L}_{h}\left(\boldsymbol{v}_{H}^{s}\right), \quad q_{h}=p_{h}^{n+1} .
\end{aligned}
$$

With this choice, from (16)-(17), one obtains:

$$
\begin{array}{r}
\rho^{f}\left(\frac{\tilde{\boldsymbol{u}}_{h}^{n+1}-\boldsymbol{u}_{h}^{n}}{\delta t}, \tilde{\boldsymbol{u}}_{h}^{n+1}\right)_{\Omega^{f}}+2 \mu\left\|\boldsymbol{\epsilon}\left(\tilde{\boldsymbol{u}}_{h}^{n+1}\right)\right\|_{0, \Omega^{f}}^{2}+\rho^{f}\left(\frac{\boldsymbol{u}^{n+1}-\tilde{\boldsymbol{u}}_{h}^{n+1}}{\delta t}, \boldsymbol{u}_{h}^{n+1}\right)_{\Omega^{f}} \\
+\rho^{s}\left(\frac{\boldsymbol{\eta}_{H}^{n+1}-2 \boldsymbol{\eta}_{H}^{n}+\boldsymbol{\eta}_{H}^{n-1}}{\delta t^{2}}, \frac{\boldsymbol{\eta}_{H}^{n+1}-\boldsymbol{\eta}_{H}^{n}}{\delta t}\right)_{\Omega^{s}}+a^{s}\left(\boldsymbol{\eta}_{H}^{n+1}, \frac{\boldsymbol{\eta}_{H}^{n+1}-\boldsymbol{\eta}_{H}^{n}}{\delta t}\right) \\
=\left\langle\mathcal{R}_{\mu}\left(\tilde{\boldsymbol{u}}_{h}^{n+1}\right), \mathcal{L}_{h}\left(\frac{\boldsymbol{\eta}_{H}^{n+1}-2 \boldsymbol{\eta}_{H}^{n}+\boldsymbol{\eta}_{H}^{n-1}}{\delta t}\right)\right\rangle .
\end{array}
$$

The residual term in the right hand-side of (21) comes from the explicit coupling of the diffusion step (16). It corresponds to the discrete counterpart of the artificial interface power

$$
\int_{\Sigma} 2 \mu \boldsymbol{\epsilon}\left(\tilde{\boldsymbol{u}}^{n+1}\right) \cdot \boldsymbol{n}^{f} \cdot\left(\tilde{\boldsymbol{u}}^{n+1}-\frac{\boldsymbol{\eta}^{n+1}-\boldsymbol{\eta}^{n}}{\delta t}\right) .
$$

Unlike fully explicit coupling (or weak coupling) this artificial term is only viscous power. Indeed, the fluid pressure is implicitly treated in step (17), so as to keep implicit the main part of the added mass effect.

Using the identity $(a-b, a)=\frac{1}{2}\|a\|^{2}-\frac{1}{2}\|b\|^{2}+\frac{1}{2}\|a-b\|^{2}$, the definition of $\mathcal{R}_{\mu}$ and the bi-linearity and symmetry of $a^{s}$, from (21), we have

$$
\begin{aligned}
& \frac{\rho^{f}}{2 \delta t}\left[\left\|\tilde{\boldsymbol{u}}_{h}^{n+1}\right\|_{0, \Omega^{f}}^{2}-\left\|\boldsymbol{u}_{h}^{n}\right\|_{0, \Omega^{f}}^{2}+\left\|\tilde{\boldsymbol{u}}_{h}^{n+1}-\boldsymbol{u}_{h}^{n}\right\|_{0, \Omega^{f}}^{2}\right]+2 \mu\left\|\boldsymbol{\epsilon}\left(\tilde{\boldsymbol{u}}_{h}^{n+1}\right)\right\|_{0, \Omega^{f}}^{2} \\
& \quad+\frac{\rho^{f}}{2 \delta t}\left[\left\|\boldsymbol{u}_{h}^{n+1}\right\|_{0, \Omega^{f}}^{2}-\left\|\tilde{\boldsymbol{u}}_{h}^{n+1}\right\|_{0, \Omega^{f}}^{2}+\left\|\boldsymbol{u}_{h}^{n+1}-\tilde{\boldsymbol{u}}_{h}^{n+1}\right\|_{0, \Omega^{f}}^{2}\right] \\
& \quad+\frac{\rho^{s}}{2 \delta t}\left[\left\|\frac{\boldsymbol{\eta}_{H}^{n+1}-\boldsymbol{\eta}_{H}^{n}}{\delta t}\right\|_{0, \Omega^{s}}^{2}-\left\|\frac{\boldsymbol{\eta}_{H}^{n}-\boldsymbol{\eta}_{H}^{n-1}}{\delta t}\right\|_{0, \Omega^{s}}^{2}+\left\|\frac{\boldsymbol{\eta}_{H}^{n+1}-2 \boldsymbol{\eta}_{H}^{n}+\boldsymbol{\eta}_{H}^{n-1}}{\delta t}\right\|_{0, \Omega^{s}}^{2}\right] \\
& \quad+\frac{1}{2 \delta t}\left[a^{s}\left(\boldsymbol{\eta}_{H}^{n+1}, \boldsymbol{\eta}_{H}^{n+1}\right)-a^{s}\left(\boldsymbol{\eta}_{H}^{n}, \boldsymbol{\eta}_{H}^{n}\right)+a^{s}\left(\boldsymbol{\eta}_{H}^{n+1}-\boldsymbol{\eta}_{H}^{n}, \boldsymbol{\eta}_{H}^{n+1}-\boldsymbol{\eta}_{H}^{n}\right)\right] \\
& =\frac{\rho^{f}}{\delta t}\left(\tilde{\boldsymbol{u}}_{h}^{n+1}-\boldsymbol{u}_{h}^{n}, \mathcal{L}_{h}\left(\frac{\boldsymbol{\eta}_{H}^{n+1}-2 \boldsymbol{\eta}_{H}^{n}+\boldsymbol{\eta}_{H}^{n-1}}{\delta t}\right)\right)_{\Omega^{f}} \\
& \quad+2 \mu\left(\boldsymbol{\epsilon}\left(\tilde{\boldsymbol{u}}_{h}^{n+1}\right), \boldsymbol{\epsilon}\left(\mathcal{L}_{h}\left(\frac{\boldsymbol{\eta}_{H}^{n+1}-2 \boldsymbol{\eta}_{H}^{n}+\boldsymbol{\eta}_{H}^{n-1}}{\delta t}\right)\right)_{\Omega^{f}} .\right.
\end{aligned}
$$


Therefore, using the coercivity of $a^{s}$ and the Cauchy-Schwarz inequality, it follows that

$$
\begin{aligned}
& \frac{\rho^{f}}{2 \delta t}\left[\left\|\boldsymbol{u}_{h}^{n+1}\right\|_{0, \Omega^{f}}^{2}-\left\|\boldsymbol{u}_{h}^{n}\right\|_{0, \Omega^{f}}^{2}\right]+\frac{\rho^{f}}{2 \delta t}\left\|\tilde{\boldsymbol{u}}_{h}^{n+1}-\boldsymbol{u}_{h}^{n}\right\|_{0, \Omega^{f}}^{2} \\
& \quad+\frac{\rho^{s}}{2 \delta t}\left[\left\|\frac{\boldsymbol{\eta}_{H}^{n+1}-\boldsymbol{\eta}_{H}^{n}}{\delta t}\right\|_{0, \Omega^{s}}^{2}-\left\|\frac{\boldsymbol{\eta}_{H}^{n}-\boldsymbol{\eta}_{H}^{n-1}}{\delta t}\right\|_{0, \Omega^{s}}^{2}+\left\|\frac{\boldsymbol{\eta}_{H}^{n+1}-2 \boldsymbol{\eta}_{H}^{n}+\boldsymbol{\eta}_{H}^{n-1}}{\delta t}\right\|_{0, \Omega^{s}}^{2}\right] \\
& \quad+\frac{1}{2 \delta t}\left[a^{s}\left(\boldsymbol{\eta}_{H}^{n+1}, \boldsymbol{\eta}_{H}^{n+1}\right)-a^{s}\left(\boldsymbol{\eta}_{H}^{n}, \boldsymbol{\eta}_{H}^{n}\right)\right]+2 \mu\left\|\boldsymbol{\epsilon}\left(\tilde{\boldsymbol{u}}_{h}^{n+1}\right)\right\|_{0, \Omega^{f}}^{2} \\
& \leq \frac{\rho^{f}}{2 \delta t}\left\|\tilde{\boldsymbol{u}}_{h}^{n+1}-\boldsymbol{u}_{h}^{n}\right\|_{0, \Omega^{f}}^{2}+\frac{\rho^{f}}{2 \delta t}\left\|\mathcal{L}_{h}\left(\frac{\boldsymbol{\eta}_{H}^{n+1}-2 \boldsymbol{\eta}_{H}^{n}+\boldsymbol{\eta}_{H}^{n-1}}{\delta t}\right)\right\|_{0, \Omega^{f}}^{2} \\
& \quad+\mu\left\|\boldsymbol{\epsilon}\left(\tilde{\boldsymbol{u}}_{h}^{n+1}\right)\right\|_{0, \Omega^{f}}^{2}+\mu\left\|\boldsymbol{\epsilon}\left(\mathcal{L}_{h}\left(\frac{\boldsymbol{\eta}_{H}^{n+1}-2 \boldsymbol{\eta}_{H}^{n}+\boldsymbol{\eta}_{H}^{n-1}}{\delta t}\right)\right)\right\|_{0, \Omega^{f}}^{2} .
\end{aligned}
$$

The following lemma (whose proof can be found in appendix), provides an estimation of $\left\|\mathcal{L}_{h}\left(\frac{\boldsymbol{\eta}_{H}^{n+1}-2 \boldsymbol{\eta}_{H}^{n}+\boldsymbol{\eta}_{H}^{n-1}}{\delta t}\right)\right\|_{0, \Omega^{f}}^{2}$ and $\left\|\boldsymbol{\epsilon}\left(\mathcal{L}_{h}\left(\frac{\boldsymbol{\eta}_{H}^{n+1}-2 \boldsymbol{\eta}_{H}^{n}+\boldsymbol{\eta}_{H}^{n-1}}{\delta t}\right)\right)\right\|_{0, \Omega^{f}}^{2}$ in terms of $h, H$ and $\left\|\frac{\boldsymbol{\eta}_{H}^{n+1}-2 \boldsymbol{\eta}_{H}^{n}+\boldsymbol{\eta}_{H}^{n-1}}{\Delta t}\right\|_{0, \Omega^{s}}^{2}$.

Lemma 1 Under the hypothesis of Theorem 1, there exists a constant $C>0$, depending only on the local mesh geometry and on the polynomial order, such that:

$$
\begin{aligned}
\left\|\mathcal{L}_{h}\left(\boldsymbol{b}_{H}\right)\right\|_{0, \Omega^{f}}^{2} & \leq \frac{C h}{H^{\alpha}}\left\|\boldsymbol{b}_{H}\right\|_{0, \Omega^{s}}^{2}, \\
\left\|\nabla \mathcal{L}_{h}\left(\boldsymbol{b}_{H}\right)\right\|_{0, \Omega^{f}}^{2} & \leq \frac{C}{h H^{\alpha}}\left\|\boldsymbol{b}_{H}\right\|_{0, \Omega^{s}}^{2},
\end{aligned}
$$

for all $\boldsymbol{b}_{H} \in V_{H}^{s}$, and $\alpha$ given by (19).

Thanks to the previous lemma we have

$$
\begin{gathered}
\left\|\mathcal{L}_{h}\left(\frac{\boldsymbol{\eta}_{H}^{n+1}-2 \boldsymbol{\eta}_{H}^{n}+\boldsymbol{\eta}_{H}^{n-1}}{\Delta t}\right)\right\|_{0, \Omega^{f}}^{2} \leq \frac{C h}{H^{\alpha}}\left\|\frac{\boldsymbol{\eta}_{H}^{n+1}-2 \boldsymbol{\eta}_{H}^{n}+\boldsymbol{\eta}_{H}^{n-1}}{\Delta t}\right\|_{0, \Omega^{s}}^{2}, \\
\left\|\boldsymbol{\epsilon}\left(\mathcal{L}_{h}\left(\frac{\boldsymbol{\eta}_{H}^{n+1}-2 \boldsymbol{\eta}_{H}^{n}+\boldsymbol{\eta}_{H}^{n-1}}{\Delta t}\right)\right)\right\|_{0, \Omega^{f}}^{2} \leq \frac{C}{h H^{\alpha}}\left\|\frac{\boldsymbol{\eta}_{H}^{n+1}-2 \boldsymbol{\eta}_{H}^{n}+\boldsymbol{\eta}_{H}^{n-1}}{\Delta t}\right\|_{0, \Omega^{s}}^{2} .
\end{gathered}
$$


Therefore, from (22), we have

$$
\begin{aligned}
& \frac{\rho^{f}}{2 \delta t}\left[\left\|\boldsymbol{u}_{h}^{n+1}\right\|_{0, \Omega^{f}}^{2}-\left\|\boldsymbol{u}_{h}^{n}\right\|_{0, \Omega^{f}}^{2}\right]+\frac{\rho^{f}}{2 \delta t}\left\|\tilde{\boldsymbol{u}}_{h}^{n+1}-\boldsymbol{u}_{h}^{n}\right\|_{0, \Omega^{f}}^{2} \\
& \quad+\frac{\rho^{s}}{2 \delta t}\left[\left\|\frac{\boldsymbol{\eta}_{H}^{n+1}-\boldsymbol{\eta}_{H}^{n}}{\delta t}\right\|_{0, \Omega^{s}}^{2}-\left\|\frac{\boldsymbol{\eta}_{H}^{n}-\boldsymbol{\eta}_{H}^{n-1}}{\delta t}\right\|_{0, \Omega^{s}}^{2}+\left\|\frac{\boldsymbol{\eta}_{H}^{n+1}-2 \boldsymbol{\eta}_{H}^{n}+\boldsymbol{\eta}_{H}^{n-1}}{\delta t}\right\|_{0, \Omega^{s}}^{2}\right] \\
& \quad+\frac{1}{2 \delta t}\left[a^{s}\left(\boldsymbol{\eta}_{H}^{n+1}, \boldsymbol{\eta}_{H}^{n+1}\right)-a^{s}\left(\boldsymbol{\eta}_{H}^{n}, \boldsymbol{\eta}_{H}^{n}\right)\right]+2 \mu\left\|\boldsymbol{\epsilon}\left(\tilde{\boldsymbol{u}}_{h}^{n+1}\right)\right\|_{0, \Omega^{f}}^{2} \\
& \leq \frac{\rho^{f}}{2 \delta t}\left\|\tilde{\boldsymbol{u}}_{h}^{n+1}-\boldsymbol{u}_{h}^{n}\right\|_{0, \Omega^{f}}^{2}+C\left(\frac{\rho^{f}}{2 \delta t} \frac{h}{H^{\alpha}}+\frac{\mu}{h H^{\alpha}}\right)\left\|\frac{\boldsymbol{\eta}_{H}^{n+1}-2 \boldsymbol{\eta}_{H}^{n}+\boldsymbol{\eta}_{H}^{n-1}}{\delta t}\right\|_{0, \Omega^{s}}^{2} \\
& \quad+\mu\left\|\boldsymbol{\epsilon}\left(\tilde{\boldsymbol{u}}_{h}^{n+1}\right)\right\|_{0, \Omega^{f}}^{2} .
\end{aligned}
$$

It follows then that

$$
\begin{array}{r}
\frac{\rho^{f}}{2 \delta t}\left[\left\|\boldsymbol{u}_{h}^{n+1}\right\|_{0, \Omega^{f}}^{2}-\left\|\boldsymbol{u}_{h}^{n}\right\|_{0, \Omega^{f}}^{2}\right]+\frac{\rho^{s}}{2 \delta t}\left[\left\|\frac{\boldsymbol{\eta}_{H}^{n+1}-\boldsymbol{\eta}_{H}^{n}}{\delta t}\right\|_{0, \Omega^{f}}^{2}-\left\|\frac{\boldsymbol{\eta}_{H}^{n}-\boldsymbol{\eta}_{H}^{n-1}}{\delta t}\right\|_{0, \Omega^{f}}^{2}\right] \\
+\frac{1}{2 \delta t}\left[a^{s}\left(\boldsymbol{\eta}_{H}^{n+1}, \boldsymbol{\eta}_{H}^{n+1}\right)-a^{s}\left(\boldsymbol{\eta}_{H}^{n}, \boldsymbol{\eta}_{H}^{n}\right)\right]+\mu\left\|\boldsymbol{\epsilon}\left(\tilde{\boldsymbol{u}}_{h}^{n+1}\right)\right\|_{0, \Omega^{f}}^{2} \\
\leq\left[C\left(\frac{\rho^{f}}{2 \delta t} \frac{h}{H^{\alpha}}+\frac{\mu}{h H^{\alpha}}\right)-\frac{\rho^{s}}{2 \delta t}\right]\left\|\frac{\boldsymbol{\eta}_{H}^{n+1}-2 \boldsymbol{\eta}_{H}^{n}+\boldsymbol{\eta}_{H}^{n-1}}{\delta t}\right\|_{0, \Omega^{s}}^{2} .
\end{array}
$$

This last inequality combined with condition (19) gives the discrete energy inequality (20), which completes the proof. $\square$

Remark 5 Theorem 1 gives a sufficient condition of stability, in the energy norm, for the semi-implicit scheme (16)-(17). Nevertheless, as mentioned above, numerical experiments show that this condition is not necessary. As a matter of fact, using (35), the trace theorem, the coercivity of $a^{s}$ and the continuous embedding $V^{s} \hookrightarrow\left[H^{1}\left(\Omega^{s}\right)\right]^{d}$, we might derive the following sufficient stability condition:

$$
\nu^{s} \geq C\left(\rho^{f} \frac{h}{\delta t^{2}}+\frac{\mu}{h \delta t}\right)
$$

with $\nu^{s}$ standing for the coercivity constant of $a^{s}$ in $V^{s}$. Although this condition prevents $h$ and $\delta t$ to tend to zero, typically $\nu^{s}$ takes large values, which gives a large range of stability. Notably, this condition ensures that (under a suitable choice of the discrete parameters $\delta t$ and $h$ ) the semi-implicit coupling scheme is stable irrespectively of the mass ratio $\rho^{s} / \rho^{f}$. As mentioned in Section 2, this property is not featured by explicit coupling schemes, see [3]. Still, by combining (19) and (24) we might also derive two new sufficient stability conditions:

$$
\rho^{s} \geq C \rho^{f} \frac{h}{H^{\alpha}}, \quad \nu^{s} \geq C \frac{\mu}{h \delta t},
$$


and

$$
\rho^{s} \geq C \mu \frac{\delta t}{h H^{\alpha}}, \quad \nu^{s} \geq C \rho^{f} \frac{h}{\delta t^{2}} .
$$

with $\alpha$ defined as in Theorem 1.

Remark 6 The $1 D$ counterpart of the the stability conditions (19) and (24)-(26), with $\alpha=1$, can be recovered numerically by explicitly coupling the wave equation (the structure) with the heat equation (the fluid). Obviously this is not surprising, since the stability conditions of our semi-implicit scheme are related to the explicit coupling of the diffusion sub-step to the structure. In this simple case the constants $C$ can be straightforwardly computed. The $1 D$ hyperbolic-parabolic explicit coupling is stable under the above conditions. In addition, numerical experiments in $1 D$ showed that the combination of these conditions seems to provide a global necessary stability condition.

Remark 7 Less restrictive stability conditions can be derived by adding a visco-elastic term to the structure equation. In particular, when dealing with a linear visco-elastic beam (see Paragraph 6.1), the semi-implicit coupling scheme is stable provided that the time step is small enough.

\section{NUMERICAL EXPERIMENTS}

In this section we illustrate the stability and efficiency features of the coupling scheme introduced in Section 4.

\subsection{Backward facing step with an elastic bottom}

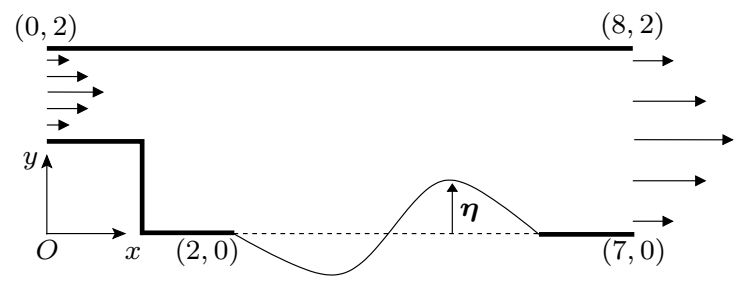

Figure 4: Backward facing step with an elastic bottom.

We consider the backward facing step with an elastic bottom wall $\Sigma$ (Figure 4). The wall displacement $\boldsymbol{\eta}$ is supposed to be along the $O y$ axis, and its vertical component, $\eta$, governed by the following equation

$$
\rho^{s} \varepsilon \frac{\partial^{2} \eta}{\partial t^{2}}-\alpha \frac{\partial^{2} \eta}{\partial z^{2}}+\beta \eta-\gamma \frac{\partial^{3} \eta}{\partial z^{2} \partial t}=\boldsymbol{f}_{\Sigma} \cdot \boldsymbol{e}_{y}
$$

where $\boldsymbol{f}_{\Sigma}$ denotes the fluid load (pressure and viscous effects) on $\Sigma, \varepsilon$ stands for the structure thickness and the coefficients $\alpha, \beta$ and $\gamma$ are given constitutive constants. This model is purely 
illustrative and we do not discuss here its mechanical relevance. The values used in our simulations were $\alpha=16.7, \beta=26.7, \gamma=0.1$. The product $\rho^{s} \varepsilon$ is either 20 or 30 depending on the test case. The fluid is governed by the incompressible Navier-Stokes equations (2) with $\rho^{f}=1, \mu=0.01$. We impose the velocity $\boldsymbol{u}=\left(u_{x}, 0\right)$, with

$$
u_{x}(y, t)=\frac{1}{4}\left(1-\cos \left(\frac{\pi t}{2}\right)\right)(y-1)(2-y)
$$

on the inlet $(x=0)$, homogeneous Neumann boundary conditions on the outlet $(x=8)$, and a no-slip boundary condition anywhere else. The time step is $\delta t=0.05$. The fluid equations are discretized using $\mathbb{Q}_{2} / \mathbb{Q}_{1}$ finite elements (1600 velocity nodes), and the structure with $\mathbb{P}_{1}$ finite elements (50 nodes). Figure 5 shows an example of the velocity and pressure fields at two different time step.
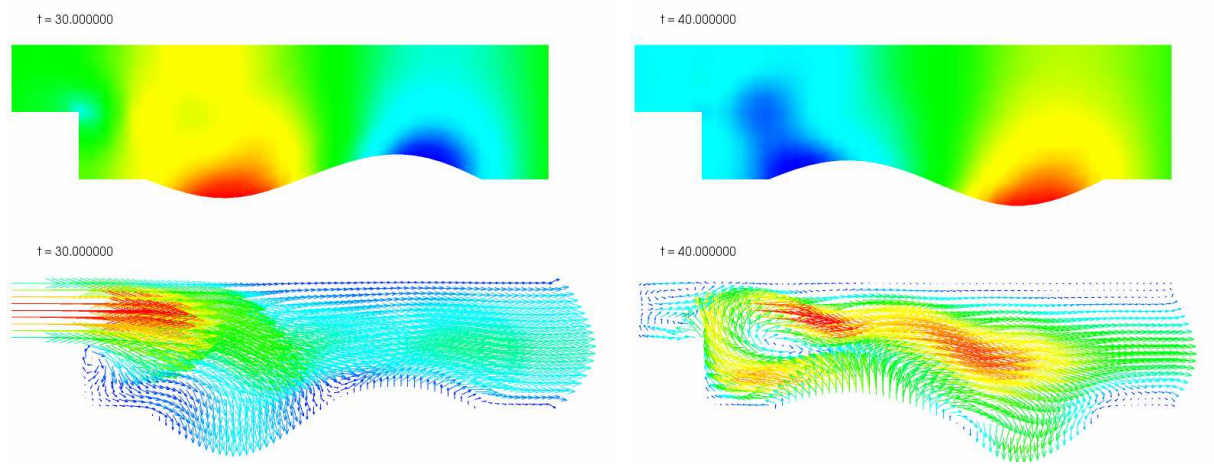

Figure 5: Velocity and pressure fields at time $t=30$ and $t=40$.

We compare the projection scheme (5)-(8) with a fully implicit coupling (solved through inexact Newton iterations [26]), and a staggered scheme (with a second order prediction of the interface position). We monitor the power transmitted through the fluid-structure interface by the fluid and by the structure at the end of each time step. Figure 6 shows the sum of these two quantities (which represents a spurious numerical power) versus time in a semi-log scale. The staggered scheme is stable for $\rho^{s} \varepsilon=30$ (Fig. 6-Left). But it generates too much spurious power and is unstable whenever the added-mass effect increases, for example, for $\rho^{s} \varepsilon=20$ (Fig. 6-Right). For the implicit and semi-implicit schemes, the spurious power is commensurate to $10^{-5}$, which is the tolerance used for the iterative algorithms. Fig. 7 points out that the interface powers obtained with the fully implicit and semi-implicit schemes are very close.

\subsection{Pressure wave in a straight cylindrical vessel}

In this paragraph we investigate the efficiency of our semi-implicit coupling scheme on the benchmark proposed in [24]. The fluid domain is a straight cylinder of radius $0.5 \mathrm{~cm}$ and length $5 \mathrm{~cm}$. The displacement of wall is described using a non-linear shell model (based 

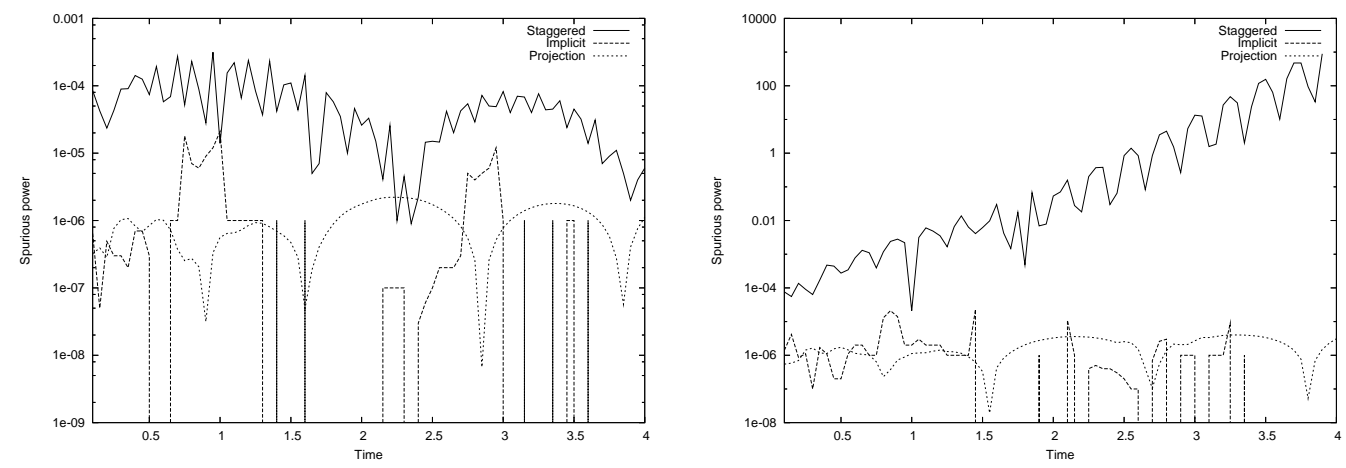

Figure 6: Left: Spurious interface power (log scale) versus time for $\rho^{s} \varepsilon=30$. Right: Spurious interface power $\left(\log\right.$ scale) versus time for $\rho^{s} \varepsilon=20$. The staggered scheme generates too much spurious power and is unstable in this case, while projection and implicit schemes remain stable.

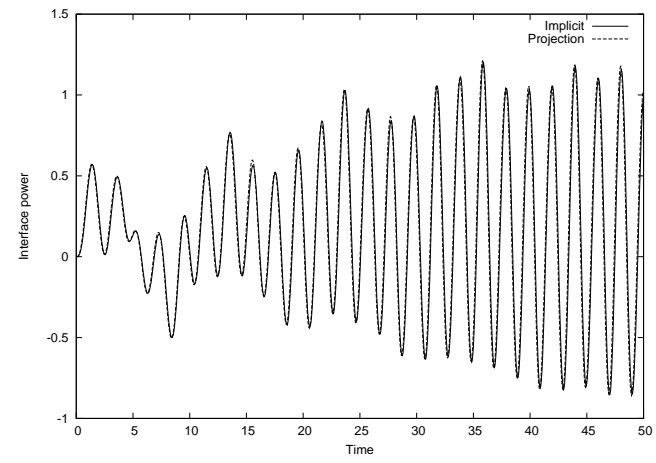

Figure 7: Power transmitted across the fluid-structure interface versus time for $\rho^{s} \varepsilon=20$. Observe the very good fitting between the projection and implicit schemes.

on MITC4 shell elements [4]). The physical parameters are: $\mu=0.03$ poise, $\rho^{f}=1 \mathrm{~g} / \mathrm{cm}^{3}$, $\rho^{s}=1.2 \mathrm{~g} / \mathrm{cm}^{3}, E=3 \times 10^{6}$ dynes $/ \mathrm{cm}^{2}, \nu=0.3$. The thickness of the vessel wall is $0.1 \mathrm{~cm}$. The fluid is initially at rest and a pressure of $1.332 \times 10^{4}$ dynes $/ \mathrm{cm}^{2}(10 \mathrm{mmHg})$ is imposed on the inlet boundary during $0.005 s$.

We use the semi-implicit scheme (5)-(8) to perform a simulation over 50 time steps of length $\delta t=0.0002 s$. The fluid part of the projection sub-step (7) is treated as a pressure Poisson problem (see [30]). The fluid equations are discretized in space using $\mathbb{Q}_{1} / \mathbb{Q}_{1}$ finite elements. The implicit sub-step (7)-(8) is solved through Newton's iterations.

A pressure wave propagation is observed. In Figure 8 we displayed the pressure at three different time steps. These results are in agreement with those reported in [24, 26, 22].

Remark 8 Although the pair $\mathbb{Q}_{1} / \mathbb{Q}_{1}$ fails to satisfy an inf-sup condition, the numerical results (Figure 8) show that the pressure approximations are stable. This is due to the intrinsic stabilizing effect of the pressure Poisson problem, provided that $\delta t$ is not too small (see [30]). 

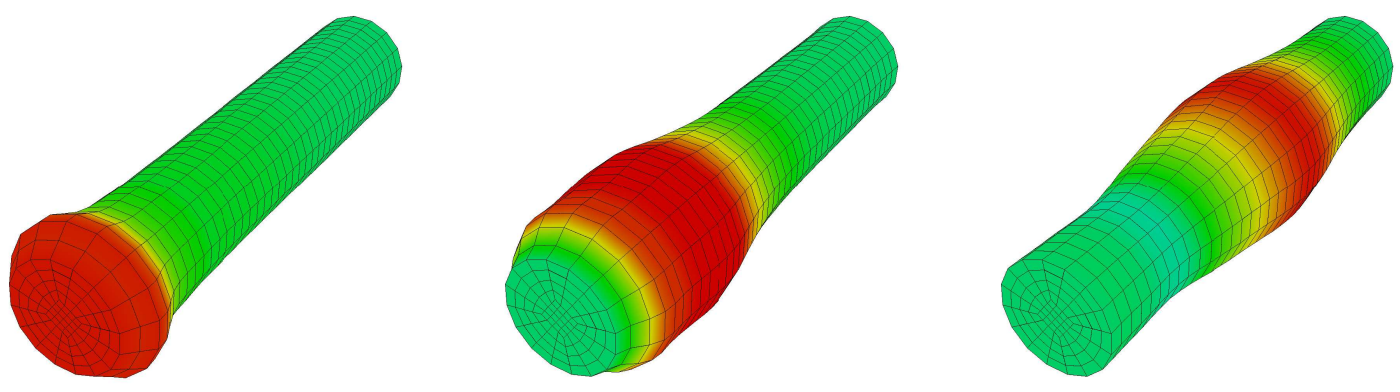

Figure 8: Pressure wave propagation in a straight vessel, $t=0.0018,0.0058$ and $0.0098 s$.

In order to point out the efficiency of the semi-implicit coupling, we reproduce the same simulation using a fully implicit coupled scheme, solved through three different algorithms:

- Aitken's accelerated fixed-point iterations (FP-Aitken), see [38, 37],

- Newton iterations using exact Jacobians (Newton), as proposed in [19, 22],

- inexact-Newton iterations (quasi-Newton) based on a reduced linear model, see [26].

The fluid equations are discretized in time using a semi-implicit scheme (coupled velocitypressure formulation) and, in space, using $\mathbb{Q}_{1} / \mathbb{Q}_{1}$ stabilized finite elements.

We furnish in Table 1 the elapsed CPU time (in seconds and dimensionless) for either approach. We can notice that the semi-implicit coupling is 4.7 times faster than the best implicit coupling (solved through Newton based iterations). This performance rises much more, a factor of 7.3, in terms of the cost of the fluid solver. Obviously, the overall computational cost reduction depends on the efficiency of the solid solver.

\begin{tabular}{|c|c|c|c|c|c|}
\hline COUPLING & ALGORITHM & $\begin{array}{c}\text { CPU } \\
\text { FLUID }\end{array}$ & $\begin{array}{c}\text { CPU } \\
\text { SOLID }\end{array}$ & $\begin{array}{c}\text { CPU } \\
\text { TOTAL }\end{array}$ & $\begin{array}{l}\text { CPU TOTAL } \\
\text { (dimensionless) }\end{array}$ \\
\hline \multirow{3}{*}{ Implicit } & FP-Aitken & 9449.43 & 2396.82 & 11846.25 & 24.86 \\
\hline & quasi-Newton & 2399.68 & 485.01 & 2884.69 & 6.05 \\
\hline & Newton & 2092.13 & 181.39 & 2273.52 & 4.77 \\
\hline Semi-Implicit & Newton & 284.20 & 192.26 & 476.46 & 1 \\
\hline
\end{tabular}

Table 1: Elapsed CPU time (in seconds and dimensionless): straight cylinder, 50 time steps of length $\delta t=0.0002 s$.

In general, the computational cost reduction observed in Table 1, with the semi-implicit coupling, is due to: (i) the use of a fractional-step method in the fluid; (ii) the explicit treatment of the ALE-advection-viscous sub-step and (iii) the use of Newton iterations for solving the projection sub-step (7)-(8). Compared to the implicit case, these iterations are 
cheap, since they do not require the computation of shape (domain) derivatives (see [22]). Numerical experiments show that solving the projection sub-step via fixed-point iterations, might lead to an algorithm which is slower than a implicit coupling solved with Newton.

\subsection{Pressure wave in a cerebral aneurysm}

We now investigate the ability of our algorithm for solving a more complex version of the previous test case, proposed in [27]. The computational domain is a cerebral aneurysm. The meshes of the fluid and solid domains are displayed in Figure 9 and are similar to those used in [27], as well as for the physical parameters: $\mu=0.06$ poise, $\rho^{f}=1 \mathrm{~g} / \mathrm{cm}^{3}, \rho^{s}=1.2 \mathrm{~g} / \mathrm{cm}^{3}$, $E=3 \times 10^{6}$ dynes $/ \mathrm{cm}^{2}, \nu=0.3$. The thickness of the vessel wall is $0.1 \mathrm{~cm}$. The fluid is initially at rest and a pressure of $10^{4}$ dynes $/ \mathrm{cm}^{2}$ is imposed on the inlet boundary during $0.005 s$.
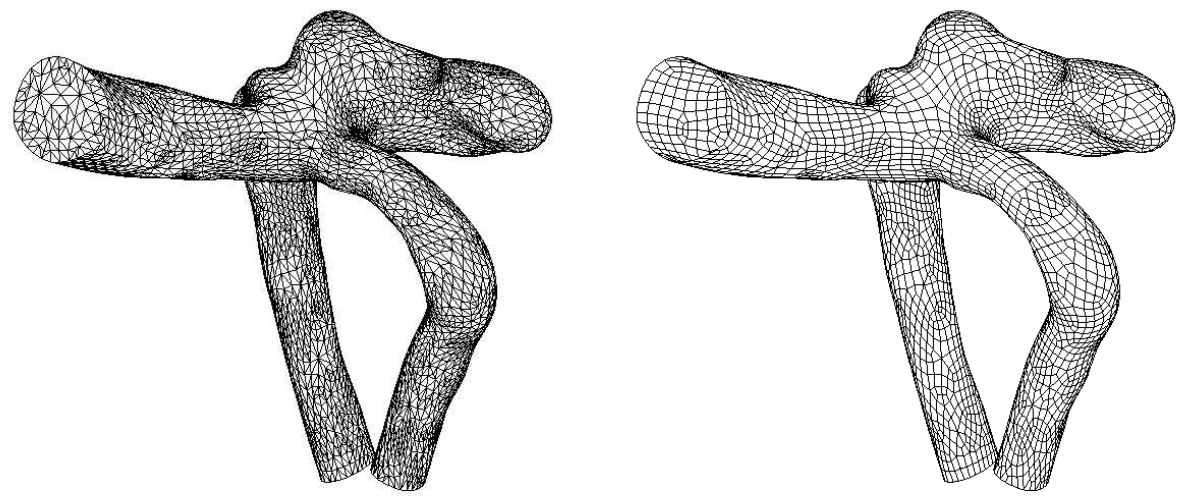

Figure 9: Fluid and solid meshes of a cerebral aneurysm: 69754 tetrahedrons and 5534 quadrilaterals.

We use the semi-implicit scheme (5)-(8) to perform a simulation over 20 time steps of length $\delta t=0.001 \mathrm{~s}$. The fluid part of the projection sub-step (7) is treated again as a pressure Poisson problem. The fluid equations are discretized in space using $\mathbb{P}_{1} / \mathbb{P}_{1}$ finite elements. A pressure wave propagation is observed. Figure 10 shows the pressure at four different time steps. These results are in agreement with those reported in [27].

Now, in order to point out the net computational gain due to use of the semi-implicit coupling, we reproduce the same simulation using a fully implicit coupled scheme with a fractional-step method in the fluid. The incompressibility constraint is again enforced through a pressure Poisson equation and we use $\mathbb{P}_{1} / \mathbb{P}_{1}$ finite elements. In Table 1 we report the elapsed CPU time (in seconds and dimensionless) of the fluid solver for either coupling. We get a computational reduction factor of 4.7 . 

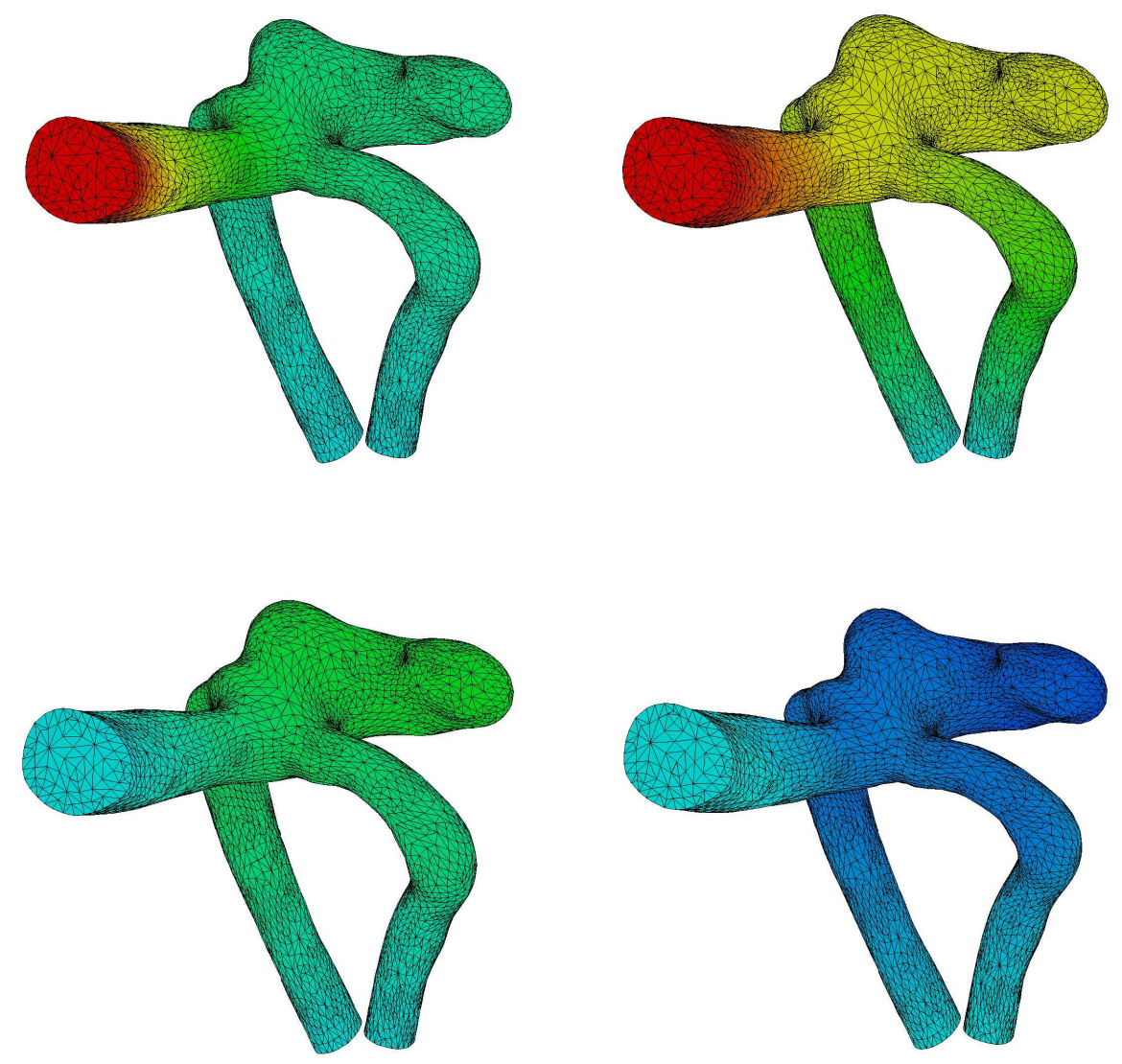

Figure 10: Pressure wave propagation in a cerebral aneurysm, $t=0.001,0.004,0.007$ and $0.014 \mathrm{~s}$.

\begin{tabular}{|c|c|c|}
\hline COUPLING & CPU & CPU FLUID \\
& FLUID & (dimensionless) \\
\hline Implicit & 2765.95 & $\mathbf{4 . 7 0}$ \\
Semi-Implicit & 587.47 & $\mathbf{1}$ \\
\hline
\end{tabular}

Table 2: Elapsed CPU time (in seconds and dimensionless): cerebral aneurysm, 20 time steps of length $\delta t=0.001 \mathrm{~s}$.

\section{CONCLUSION}

In this work we focused on the numerical simulation of fluid-structure systems involving a viscous fluid and featuring a strong added-mass effect. This arises, for instance, in the internal 
flow of an incompressible fluid whose density is close to the structure density. Blood flows are a typical example. In such situations, explicit coupling schemes appear to be unstable. Up to now, this drawback was overcome by using fully implicit coupling schemes, namely, preserving the energy balance but with the payoff of solving a coupled highly non-linear system at each time step.

The main contribution of this paper consists in introducing and analyzing a semi-implicit coupling scheme (i.e. partially explicit) which remains stable in test cases for which fully implicit schemes were the only known to be stable.

In order to ensure stability we couple implicitly the pressure stress to the structure, whereas the remaining fluid expensive terms (dissipation, convection and geometrical nonlinearities) are explicitly treated (i.e. out of the sub-iterations). The trick is that this kind of implicit-explicit splitting can be naturally performed using a Chorin-Temam projection scheme for the fluid. Thus, in short, at each time step we proposed to implicit couple the projection sub-step (carried out in a known fixed fluid domain) with the structure, while the expensive ALE-advection-viscous sub-step is explicitly coupled.

The main features of the resulting algorithm are: its simplicity of implementation and its efficiency compared to any strongly coupled schemes, we are aware of, as shown in the different numerical experiments. Finally, we provided a rigorous theoretical result ensuring the conditional stability of the scheme when applied to a linear model problem.

There exist several ways of extending the work presented in this paper. For instance, we shall perform the convergence analysis on the linear model problem. We may then address the stability analysis of the scheme in the non-linear case (i.e. with a fluid moving domain). We shall also investigate the applicability of our idea when dealing with other kind of fractionalstep methods within the fluid.

\section{ACKNOWLEDGEMENTS}

The present work has been carried out during a one-year délégation of C. Grandmont at INRIA and partially supported by the European Community through the Research Training Network "Mathematical Modelling of the Cardiovascular System (HaeMOdel)", contract HPRN-CT-2002-00270.

\section{APPENDIX}

Proof of Lemma 1. Firstly, we recall the following classical estimations concerning finite element shape functions:

$$
\begin{gathered}
\left\|\varphi_{j}^{K}\right\|_{0, K}^{2} \leq C h^{d}, \\
\left\|\nabla \varphi_{j}^{K}\right\|_{0, K}^{2} \leq C h^{d-2},
\end{gathered}
$$

for all $K \in \mathcal{T}_{h}^{f}, j=1, \ldots, N$, where $N$ stands for the number of degrees of freedom of $K$. Let us remind as well that $V_{H}^{s}(\Sigma)$ and $V_{h}^{f}(\Sigma)$ stand for the trace finite element spaces associated to $V_{H}^{s}$ and $V_{h}^{f}$, respectively. We have the following local inverse inequality (see [14]):

$$
\left\|\boldsymbol{b}_{h}\right\|_{0, \infty, K \cap \Sigma}^{2} \leq C h^{-d+1}\left\|\boldsymbol{b}_{h}\right\|_{0, K \cap \Sigma}^{2}, \quad \forall \boldsymbol{b}_{h} \in V_{h}^{f}(\Sigma),
$$


as well as the discrete trace inequality (see [7])

$$
\left\|\boldsymbol{b}_{H}\right\|_{0, \partial K}^{2} \leq C H^{-1}\left\|\boldsymbol{b}_{H}\right\|_{0, K}^{2}, \quad \forall \boldsymbol{b}_{H} \in V_{H}^{s}, \quad \forall K \in \mathcal{T}_{H}^{s}
$$

From the definition of the discrete lifting operator $\mathcal{L}_{h}(18)$, we have

$$
\begin{aligned}
& \left\|\mathcal{L}_{h}\left(\boldsymbol{b}_{H}\right)\right\|_{0, \Omega^{f}}^{2}=\sum_{\substack{K \in \mathcal{T}_{h}^{f} \\
K \cap \Sigma \neq \emptyset}}\left\|\mathcal{L}_{h}\left(\boldsymbol{b}_{H}\right)_{\mid K}\right\|_{0, K}^{2} \\
& =\sum_{\substack{K \in \mathcal{T}_{h}^{f} \\
K \cap \Sigma \neq \emptyset \\
|K \cap \Sigma| \neq 0}}\left\|\mathcal{L}_{h}\left(\boldsymbol{b}_{H}\right)_{\mid K}\right\|_{0, K}^{2}+\sum_{\substack{K \in \mathcal{T}_{h}^{f} \\
K \cap \Sigma \neq \emptyset \\
|K \cap \Sigma|=0}}\left\|\mathcal{L}_{h}\left(\boldsymbol{b}_{H}\right)_{\mid K}\right\|_{0, K}^{2},
\end{aligned}
$$

with $|K \cap \Sigma|$ standing for the $(d-1)$-Lebesgue measure of $K \cap \Sigma$.

Now we estimate separately the two sums in (30). Let $\left\{x_{j}^{K}\right\}_{j=1}^{N}$ be the set of local nodes in $K$ and $\left\{\phi_{j}^{K}\right\}_{j=1}^{N}$ their corresponding shape functions. For each $K \in \mathcal{T}_{h}^{f}$ with $K \cap \Sigma \neq \emptyset$ and $|K \cap \Sigma| \neq 0$, using (27) 1 and (28). one gets

$$
\begin{aligned}
\left\|\mathcal{L}_{h}\left(\boldsymbol{b}_{H}\right)_{\mid K}\right\|_{0, K}^{2} & =\left\|\sum_{\substack{j=1 \\
\boldsymbol{x}_{j}^{K} \in \Sigma}}^{N} \pi_{h}\left(\boldsymbol{b}_{H}\right)\left(\boldsymbol{x}_{j}^{K}\right) \varphi_{j}^{K}\right\|_{0, K}^{2} \\
& \leq \sum_{\substack{j=1 \\
\boldsymbol{x}_{j}^{K} \in \Sigma}}^{N}\left|\pi_{h}\left(\boldsymbol{b}_{H}\right)\left(\boldsymbol{x}_{j}^{K}\right)\right|^{2}\left\|\varphi_{j}^{K}\right\|_{0, K}^{2} \\
& \leq C h^{d}\left\|\pi_{h}\left(\boldsymbol{b}_{H}\right)\right\|_{0, \infty, K \cap \Sigma}^{2} \\
& \leq C h\left\|\pi_{h}\left(\boldsymbol{b}_{H}\right)\right\|_{0, K \cap \Sigma}^{2} .
\end{aligned}
$$

Finally, consider the case $K \in \mathcal{T}_{h}^{f}$ with $K \cap \Sigma \neq \emptyset$ and $|K \cap \Sigma|=0$. As in (31),

$$
\begin{aligned}
\left\|\mathcal{L}_{h}\left(\boldsymbol{b}_{H}\right)_{\mid K}\right\|_{0, K}^{2} & \leq \sum_{\substack{j=1 \\
\boldsymbol{x}_{j}^{K} \in \Sigma}}^{N}\left|\pi_{h}\left(\boldsymbol{b}_{H}\right)\left(\boldsymbol{x}_{j}^{K}\right)\right|^{2}\left\|\varphi_{j}^{K}\right\|_{0, K}^{2} \\
& \leq C h^{d} \sum_{\substack{j=1 \\
\boldsymbol{x}_{j}^{K} \in \Sigma}}^{N}\left|\pi_{h}\left(\boldsymbol{b}_{H}\right)\left(\boldsymbol{x}_{j}^{K}\right)\right|^{2} .
\end{aligned}
$$

Obviously, there exists $K^{j} \in \mathcal{T}_{h}^{f}$ with $\left|K^{j} \cap \Sigma\right| \neq 0$ and such that $\boldsymbol{x}_{j}^{K} \in K^{j}$. Therefore,

$$
\left\|\mathcal{L}_{h}\left(\boldsymbol{b}_{H}\right)_{\mid K}\right\|_{0, K}^{2} \leq C h^{d} \sum_{\substack{j=1 \\ \boldsymbol{x}_{j}^{K} \in \Sigma}}^{N}\left\|\pi_{h}\left(\boldsymbol{b}_{H}\right)\right\|_{0, \infty, K^{j} \cap \Sigma}^{2}
$$


Thus, from (30), (31) and (32), we have

$$
\begin{aligned}
&\left\|\mathcal{L}_{h}\left(\boldsymbol{b}_{H}\right)\right\|_{0, \Omega^{f}}^{2} \leq C h \sum_{\substack{K \in \mathcal{T}_{h}^{f} \\
|K \cap \Sigma| \neq 0}}\left\|\pi_{h}\left(\boldsymbol{b}_{H}\right)\right\|_{0, K \cap \Sigma}^{2} \\
&+C h^{d} \sum_{\substack{K \in \mathcal{T}_{h}^{f} \\
K \cap \Sigma \neq \emptyset \\
|K \cap \Sigma|=0}} \sum_{\substack{j=1 \\
\boldsymbol{x}_{j}^{K} \in \Sigma}}^{N}\left\|\pi_{h}\left(\boldsymbol{b}_{H}\right)\right\|_{0, \infty, K^{j} \cap \Sigma}^{2} \cdot
\end{aligned}
$$

The quasi-uniformity (15) of the fluid triangulation ensures that the number of elements containing a node $\boldsymbol{x}_{j}^{K}$ is independent of $h$. Thus, it follows that

$$
\begin{aligned}
\sum_{\substack{K \in \mathcal{T}_{h}^{f} \\
K \cap \Sigma \neq \emptyset \\
|K \cap \Sigma|=0}} \sum_{\substack{j=1 \\
\boldsymbol{x}_{j}^{K} \in \Sigma}}^{N}\left\|\pi_{h}\left(\boldsymbol{b}_{H}\right)\right\|_{0, \infty, K^{j} \cap \Sigma}^{2} \leq C \sum_{\substack{K \in \mathcal{T}_{h}^{f} \\
|K \cap \Sigma| \neq 0}}\left\|\pi_{h}\left(\boldsymbol{b}_{H}\right)\right\|_{0, \infty, K \cap \Sigma}^{2} \\
\leq C h^{-d+1} \sum_{\substack{K \in \mathcal{T}_{h}^{f} \\
|K \cap \Sigma| \neq 0}}\left\|\pi_{h}\left(\boldsymbol{b}_{H}\right)\right\|_{0, K \cap \Sigma}^{2},
\end{aligned}
$$

in the last inequality we used again (28). Therefore, from (33), we finally obtain

$$
\begin{aligned}
\left\|\mathcal{L}_{h}\left(\boldsymbol{b}_{H}\right)\right\|_{0, \Omega^{f}}^{2} & \leq C h \sum_{\substack{K \in \mathcal{T}_{h}^{f} \\
|K \cap \Sigma| \neq 0}}\left\|\pi_{h}\left(\boldsymbol{b}_{H}\right)\right\|_{0, K \cap \Sigma}^{2}, \\
& =C h\left\|\pi_{h}\left(\boldsymbol{b}_{H}\right)\right\|_{0, \Sigma}^{2} .
\end{aligned}
$$

Since $\pi_{h} L^{2}$-stable in $V_{H}^{s}(\Sigma)$, it then follows that

$$
\left\|\mathcal{L}_{h}\left(\boldsymbol{b}_{H}\right)\right\|_{0, \Omega^{f}}^{2} \leq C h\left\|\boldsymbol{b}_{H}\right\|_{0, \Sigma}^{2}
$$

which gives $(23)_{1}$ for $\alpha=0$ (i.e. $\overline{\Omega^{s}}=\Sigma$ ). For the case $\overline{\Omega^{s}} \neq \Sigma$, we combine (35) with the discrete trace inequality (29). This yields

$$
\begin{aligned}
\left\|\mathcal{L}_{h}\left(\boldsymbol{b}_{H}\right)\right\|_{0, \Omega^{f}}^{2} & \leq C h\left\|\boldsymbol{b}_{H}\right\|_{0, \Sigma}^{2} \\
& =C h \sum_{\substack{K \in \mathcal{T}_{H}^{s} \\
K \cap \Sigma \neq \emptyset}}\left\|\boldsymbol{b}_{H}\right\|_{0, K \cap \Sigma}^{2} \\
& \leq C \frac{h}{H} \sum_{\substack{K \in \mathcal{T}_{H}^{s} \\
K \cap \Sigma \neq \emptyset}}\left\|\boldsymbol{b}_{H}\right\|_{0, K}^{2} \\
& \leq C \frac{h}{H}\left\|\boldsymbol{b}_{H}\right\|_{0, \Omega^{s}}^{2},
\end{aligned}
$$

which provides $(23)_{1}$ with $\alpha=1$. Finally, $(23)_{2}$ follows using a similar argument in combination with $(27)_{2}$, which completes the proof of the lemma. 
Lemma 2 Let $\pi_{h}: V_{H}^{s}(\Sigma) \longrightarrow V_{h}^{f}(\Sigma)$ be the standard Lagrange finite element interpolation operator, and assume that fluid interface mesh is a sub-triangulation of the solid interface mesh, namely, for all $K \in \mathcal{T}_{h}^{f}$ with $|K \cap \Sigma| \neq 0$ there exists $K^{\prime} \in \mathcal{T}_{H}^{s}$ such that

$$
K \cap \Sigma \subset K^{\prime} \cap \Sigma \text {. }
$$

Then, $\pi_{h}$ is $L^{2}$-stable in $V_{H}^{s}(\Sigma)$.

Proof. For each $\boldsymbol{b}_{H} \in V_{H}^{s}$, using $(27)_{1}$ (on the interface elements) and the definition of the Lagrange finite element interpolation operator, we have

$$
\begin{aligned}
&\left\|\pi_{h}\left(\boldsymbol{b}_{H}\right)\right\|_{0, \Sigma}^{2} \sum_{\substack{K \in \mathcal{T}_{h}^{f} \\
|K \cap \Sigma| \neq \emptyset}}\left\|\pi_{h}\left(\boldsymbol{b}_{H}\right)\right\|_{0, K \cap \Sigma}^{2} \\
& \leq C h^{d-1} \sum_{\substack{K \in \mathcal{T}_{h}^{f} \\
|K \cap \Sigma| \neq \emptyset}}\left\|\boldsymbol{b}_{H}\right\|_{0, \infty, K \cap \Sigma}^{2} .
\end{aligned}
$$

In addition, from (36), the restriction of $\boldsymbol{b}_{H}$ to any $K \cap \Sigma$ (with $K \in \mathcal{T}_{h}^{f}$ and $|K \cap \Sigma| \neq \emptyset$ ) is a polynomial function. Thus, the following local inverse inequality holds true:

$$
\left\|\boldsymbol{b}_{H}\right\|_{0, \infty, K \cap \Sigma}^{2} \leq C h^{-d+1}\left\|\boldsymbol{b}_{H}\right\|_{0, K \cap \Sigma}^{2} .
$$

As a result, from (37), we have

$$
\left\|\pi_{h}\left(\boldsymbol{b}_{H}\right)\right\|_{0, \Sigma}^{2} \leq C\left\|\boldsymbol{b}_{H}\right\|_{0, \Sigma}^{2}
$$

which completes the proof of the lemma.

\section{References}

[1] F. Baaijens. A fictitious domain/mortar element method for fluid-structure interaction. Int. Jour. Num. Meth. Fluids, 35:743-761, 2001.

[2] C. Bernardi, Y. Maday, and A. T. Patera. Domain decomposition by the mortar element method. In Asymptotic and numerical methods for partial differential equations with critical parameters (Beaune, 1992), volume 384 of NATO Adv. Sci. Inst. Ser. C Math. Phys. Sci., pages 269-286. Kluwer Acad. Publ., Dordrecht, 1993.

[3] P. Causin, J.-F. Gerbeau, and F. Nobile. Added-mass effect in the design of partitioned algorithms for fluid-structure problems. Comp. Meth. Appl. Mech. Engng., 194(42-44):4506-4527, 2005.

[4] D. Chapelle and K.J. Bathe. The Finite Element Analysis of Shells - Fundamentals. Springer Verlag, 2003.

[5] A.J. Chorin. Numerical solution of the Navier-Stokes equations. Math. Comp., 22:745-762, 1968.

[6] A.J. Chorin. On the convergence of discrete approximations to the Navier-Stokes equations. Math. Comp., 23:341-353, 1969.

[7] Ph. Clément. Approximation by finite element functions using local regularization. Rev. Française Automat. Informat. Recherche Opérationnelle Sér. RAIRO Analyse Numérique, 9(R-2):77-84, 1975. 
[8] J. De Hart, G.W.M. Peters, P.J.G. Schreurs, and F.P.T. Baaijens. A three-dimensional computational analysis of fluid-structure interaction in the aortic valve. Journal of Biomechanics, $36: 103-112,2003$.

[9] S. Deparis, M. Discacciati, G. Fourestey, and A. Quarteroni. Heterogeneous domain decomposition methods for fluid-structure interaction problems. Technical report, EPFL-IACS report $08.2005,2005$.

[10] S. Deparis, M. Discacciati, and A. Quarteroni. A domain decomposition framework for fluidstructure interaction problems. In Proceedings of the Third International Conference on Computational Fluid Dynamics (ICCFD3), 2004.

[11] S. Deparis, M.A. Fernández, and L. Formaggia. Acceleration of a fixed point algorithm for fluid-structure interaction using transpiration conditions. M2AN Math. Model. Numer. Anal., $37(4): 601-616,2003$.

[12] S. Deparis, J.-F. Gerbeau, and X. Vasseur. A Dynamic Preconditioner for Newton-Krylov Algorithms. Application to Fluid-Structure Interaction. RR INRIA No 5277, 2004.

[13] J. Donéa, S. Giuliani, and J. P. Halleux. An arbitrary Lagrangian-Eulerian finite element method for transient dynamic fluid-structure interactions. Comp. Meth. Appl. Mech. Engng., pages 689$723,1982$.

[14] A. Ern and J.-L. Guermond. Theory and practice of finite elements, volume 159 of Applied Mathematical Sciences. Springer-Verlag, New York, 2004.

[15] C. Farhat, M. Lesoinne, and P. Le Tallec. Load and motion transfer algorithms for fluid/structure interaction problems with non-matching discrete interfaces: Momentum and energy conservation, optimal discretization and application to aeroelasticity. Comput. Methods Appl. Mech. Engrg., 157:95-114, 1998.

[16] C. Farhat, K. van der Zee, and Ph. Geuzaine. Provably second-order time-accurate looselycoupled solution algorithms for transient nonlinear aeroelasticity. to appear in Comp. Meth. Appl. Mech. Engng.

[17] C.A. Felippa. Underwater shock on stiffened shells: the source of Staggered Solution Procedure. In 5th international Conference on Computational of Shell 83 Spatial Structures. IASS-IACM, 2005.

[18] C.A. Felippa and J.A. Deruntz. Finite element analysis of shock-induced hull cavitation. Comput. Methods Appl. Mech. Engrg., 44(3):297-337, 1984.

[19] M. A. Fernández and M. Moubachir. Numerical simulation of fluid-structure systems via Newton's method with exact Jacobians. In P Neittaanmäki, T. Rossi, K. Majava, and O. Pironneau, editors, $4^{\text {th }}$ European Congress on Computational Methods in Applied Sciences and Engineering, volume 1, Jyväskylä, Finland, July 2004.

[20] M.A. Fernández and M. Moubachir. An exact block-Newton algorithm for solving fluid-structure interaction problems. C. R. Math. Acad. Sci. Paris, 336(8):681-686, 2003.

[21] M.A. Fernández and M. Moubachir. An exact block-newton algorithm for the solution of implicit time discretized coupled systems involved in fluid-structure interaction problems. In K.J. Bathe, editor, Second M.I.T. Conference on Computational Fluid and Solid Mechanics, pages 1337-1341. Elsevier, 2003. 
[22] M.A. Fernández and M. Moubachir. A Newton method using exact Jacobians for solving fluidstructure coupling. Comp. ES Struct., 83:127-142, 2005.

[23] A. Figueroa, I. Vignon-Clementel, K.E. Jansen, T.J.R. Hughes, and C.A. Taylor. A coupled momentum method for modeling blood flow in three-dimensional deformable arteries. To appear in Comput. Methods Appl. Mech. Engrg.

[24] L. Formaggia, J.-F. Gerbeau, F. Nobile, and A. Quarteroni. On the coupling of 3D and 1D Navier-Stokes equations for flow problems in compliant vessels. Comp. Meth. Appl. Mech. Engrg., 191(6-7):561-582, 2001.

[25] J.-F. Gerbeau. A quasi-Newton method for a fluid-structure problem arising in blood flows. In K.J. Bathe, editor, Proceedings of the second M.I.T. Conference on Computational Fluid and Solid Mechanics, pages 1355-1357. Elsevier, 2003.

[26] J.-F. Gerbeau and M. Vidrascu. A quasi-Newton algorithm based on a reduced model for fluidstructure interactions problems in blood flows. Math. Model. Num. Anal., 37(4):631-648, 2003.

[27] J.-F. Gerbeau, M. Vidrascu, and P. Frey. Fluid-structure interaction in blood flows on geometries based on medical imaging. Comp. \& Struct., 83(2-3):155-165, 2005.

[28] C. Grandmont and Y. Maday. Nonconforming grids for the simulation of fluid-structure interaction. In Domain decomposition methods, 10 (Boulder, CO, 1997), volume 218 of Contemp. Math., pages 262-270. Amer. Math. Soc., Providence, RI, 1998.

[29] J.-L. Guermond, P. Minev, and J. Shen. An overview of projection methods for incompressible flows. Submitted to Comp. Meth. Appl. Mech. Engng., 2004.

[30] J.-L. Guermond and L. Quartapelle. On stability and convergence of projection methods based on pressure Poisson equation. Internat. J. Numer. Methods Fluids, 26(9):1039-1053, 1998.

[31] M. Heil. An efficient solver for the fully coupled solution of large-displacement fluid-structure interaction problems. Comput. Methods Appl. Mech. Engrg., 193(1-2):1-23, 2004.

[32] P. Le Tallec and J. Mouro. Fluid structure interaction with large structural displacements. Comput. Meth. Appl. Mech. Engrg., 190:3039-3067, 2001.

[33] H.G. Matthies and J. Steindorf. Partitioned but strongly coupled iteration schemes for nonlinear fluid-structure interaction. Comp. \&5 Struct., 80(27-30):1991-1999, 2002.

[34] H.G. Matthies and J. Steindorf. Strong coupling methods. Technical report, TU Braunschweig, 2002.

[35] H.G. Matthies and J. Steindorf. Partitioned strong coupling algorithms for fluid-structure interaction. Comp. \& Struct., 81:805-812, 2003.

[36] C. Michler, E.H. van Brummelen, and R. de Borst. An interface Newton-Krylov solver for fluidstructure interaction. Int. J. Num. Meth. Fluids, 47(10-11):1189-1195, 2005.

[37] D. P. Mok and W. A. Wall. Partitioned analysis schemes for the transient interaction of incompressible flows and nonlinear flexible structures. In K. Schweizerhof W.A. Wall, K.U. Bletzinger, editor, Trends in computational structural mechanics, Barcelona, 2001. CIMNE.

[38] D. P. Mok, W. A. Wall, and E. Ramm. Partitioned analysis approach for the transient, coupled response of viscous fluids and flexible structures. In W. Wunderlich, editor, Proceedings of the European Conference on Computational Mechanics. ECCM'99, TU Munich, 1999. 
[39] D. P. Mok, W. A. Wall, and E. Ramm. Accelerated iterative substructuring schemes for instationary fluid-structure interaction. In K.J. Bathe, editor, Computational Fluid and Solid Mechanics, pages 1325-1328. Elsevier, 2001.

[40] F. Nobile. Numerical approximation of fluid-structure interaction problems with application to haemodynamics. PhD thesis, EPFL, Switzerland, 2001.

[41] D. Perić and W. G. Dettmer. A computational strategy for interaction of fluid flow with spatial structures. In 5th international Conference on Computational of Shell 8 Spatial Structures. IASS-IACM, 2005.

[42] S. Piperno. Explicit/implicit fluid/structure staggered procedures with a structural predictor and fluid subcycling for 2D inviscid aeroelastic simulations. Internat. J. Numer. Methods Fluids, 25(10):1207-1226, 1997.

[43] S. Piperno, C. Farhat, and B. Larrouturou. Partitioned procedures for the transient solution of coupled aeroelastic problems. Part I: Model problem, theory and two-dimensional application. Comp. Meth. Appl. Mech. Engrg., 124:79-112, 1995.

[44] P. Raback, J. Ruokolainen, M. Lyly, and E. Järvinen. Fluid-structure interaction boundary conditions by artificial compressibility. In ECCOMAS Computational Fluid Dynamics Conference, Swansea, 2001, 2001.

[45] S. Rugonyi and K.J. Bathe. On finite element analysis of fluid flows coupled with structural interaction. CMES - Comp. Modeling Eng. Sci., 2(2):195-212, 2001.

[46] R. Temam. Une méthode d'approximation de la solution des équations de Navier-Stokes. Bull. Soc. Math. France, 96:115-152, 1968.

[47] T.E. Tezduyar. Finite element methods for fluid dynamics with moving boundaries and interfaces. Arch. Comput. Methods Engrg., 8:83-130, 2001.

[48] H. Zhang, X. Zhang, S. Ji, Y. Guo, G. Ledezma, N. Elabbasi, and H. deCougny. Recent development of fluid-structure interaction capabilities in the ADINA system. Comp. ES Struct., 81(8-11):1071-1085, 2003. 